\title{
Explosive particle creation by instantaneous change of boundary conditions
}

\author{
Umpei Miyamoto* \\ RECCS, Akita Prefectural University, Akita 015-0055, Japan
}

(Received 31 October 2018; revised manuscript received 24 December 2018; published 25 January 2019)

\begin{abstract}
We investigate the dynamic Casimir effect (DCE) of a $(1+1)$-dimensional free massless scalar field in a finite or semi-infinite cavity for which the boundary condition (BC) instantaneously changes from the Neumann to the Dirichlet BC or reversely. While this setup is motivated by the gravitational phenomena, such as the formation of strong naked singularities or wormholes, and the topology change of spacetimes or strings in quantum gravity, the analysis is quite general. For the Neumann-to-Dirichlet cases, we find two components of diverging flux emanate from the point where the $\mathrm{BC}$ changes. We carefully compare this result with that of Ishibashi and Hosoya (2002) obtained in the context of a quantum version of cosmic censorship hypothesis, and show that one of the diverging components was overlooked by them and is actually nonrenormalizable, suggesting to bring non-negligible backreaction or semiclassical instability. On the other hand, for the Dirichlet-to-Neumann cases, we reveal for the first time that only one component of diverging flux emanates, which is the same kind as that overlooked in the Neumann-to-Dirichlet cases. This result suggests not only the robustness of the appearance of diverging flux in instantaneous limits of DCE but also that the type of divergence sensitively depends on the combination of initial and final BCs.
\end{abstract}

DOI: $10.1103 /$ PhysRevD.99.025012

\section{INTRODUCTION}

One of the surprising pictures that quantum field theories provide is that a classical vacuum is fluctuating, in which virtual particles spontaneously appear and disappear in short periods of time allowed by the Heisenberg uncertainty principle. The phenomenon that neutral conductive plates put parallel in a classical electromagnetic vacuum attract each other is caused by such particles and called the Casimir effect [1] (see [2] for a review). If one moves the plates (or boundaries for a quantum field in general), the virtual particles can convert into real ones, which is known as the dynamic Casimir effect (DCE) [3] (see [4] for a review).

In general, in order to realize and detect the DCE experimentally by moving a boundary, its speed has to be accelerated up to a few percent of the speed of light. Therefore, such an experiment had been thought to be quite difficult. The effect brought by moving a boundary, however, was recognized to be realized effectively by modulating with high frequency the electromagnetic properties of a static boundary. Based on this idea, the DCE was indeed observed first by Wilson et al. [5] using a

\footnotetext{
*umpei@akita-pu.ac.jp
}

Published by the American Physical Society under the terms of the Creative Commons Attribution 4.0 International license. Further distribution of this work must maintain attribution to the author(s) and the published article's title, journal citation, and DOI. Funded by SCOAP. superconducting circuit. So far, there have been proposed various experimental methods to realize the DCE [6-8], and various theoretical results have been obtained [9].

It is mentioned that the dynamics of quantum systems undergoing a rapid change of parameter in their Hamiltonian (note that the change of boundary conditions can be included as terms in the Hamiltonian) is called the quantum quench dynamics and is actively studied nowadays since it poses many fundamental questions that can be studied by current-generation experiments [10]. For example, the effect of the time-periodic boundary condition (i.e., Floquet dynamics) in a conformal field theory, which is a low-energy description of quantum critical systems, has been investigated [11]. The entanglement entropy of a conformal field excited by the change of boundary conditions (BC) also has been studied [12].

While the DEC is a universal phenomenon caused by time-dependent $\mathrm{BCs}$, it occupies a special position in general relativity and other gravitational theories since the effects similar or equivalent to time-dependent BCs are realized not artificially but naturally in dynamical spacetimes, such as the expanding universe [13], the gravitational collapse of stars to black holes [14], the creation of naked singularities $[15,16]$ and wormholes [17], and the topology change of spacetime (and string world sheets) in quantum gravity [18-20] (see $[21,22]$ for a comprehensive study of quantum field theory in curved spacetimes).

Among the above phenomena in gravitational physics, the particle creation due to the formation of naked 
singularities [16] is of fundamental importance since it is closely related to the future predictability of the law of quantum physics, namely, the existence of a cosmic censor [23] from the quantum physics point of view. The basic idea is as follows: The spacetime singularity, in which the predictability of the law of physics is thought to be lost if the singularity is naked or visible, is defined by the geodesic incompleteness [24]. However, such a definition of singular spacetime using the notion of particles may judge a spacetime appearing harmless (e.g., Minkowski spacetime from which a single point is taken out) to be singular. Therefore, it was proposed to define the spacetime regularity with not the geodesic completeness but a uniqueness of propagation of classical wave fields (or a uniqueness of the self-adjoint extension of the time-translation operator) $[25,26]$. Such a definition using the notion of fields actually excludes the spacetimes appearing harmless from a class of singular spacetimes [27].

Ishibashi and Hosoya [16] proceeded to a next step. Namely, they investigated what happens if one quantizes a wave field in the "wave-singular" (therefore singular also in the ordinary geodesic sense) spacetime describing the formation of a strong naked singularity, which can be modeled by an instantaneous change of $\mathrm{BCs}$ for the wave field. More specifically, they considered a quantized $(1+1)$ dimensional free massless scalar field in a cavity for which the BC suddenly changes from the Neumann to the Dirichlet. They showed that a diverging flux taking the form of a delta function squared emanates from the points where the BCs change and propagates along null lines. From such a result, they concluded that the backreaction of created particles would bring null singularities, resulting in the recovery of global hyperbolicity (i.e., the future predictability of the law of physics). That is, the created particles play the role of a quantum version of the cosmic censor.

While the idea of the quantum version of the cosmic censor is interesting and shown to work in [16], an unsatisfactory point may be that the analysis was restricted to the Neumann-to-Dirichlet case. Although Ishibashi and Hosoya tried to examine a more general case for which the $\mathrm{BC}$ changes from a Robin $\mathrm{BC} \phi(t, x)=a \partial_{x} \phi$ to another Robin one $\phi(t, x)=b \partial_{x} \phi[a$ and $b(\neq a)$ are constants and both sides of the equalities are evaluated at the boundary], but failed to obtain any rigorous result. (See [28] for a systematic study on the static Casimir effect under Robin BCs and $[8,29,30]$ for the DCE with time-dependent Robin BCs with a nonrelativistic approximation.)

Therefore, in this paper, we shall extend the analysis in Ref. [16] in two directions. First, we examine the instantaneous change of $\mathrm{BC}$ in a finite cavity from the Dirichlet to Neumann. Then, we examine both the Neumann-toDirichlet (N-D) and the Dirichlet-to-Neumann (D-N) cases in a semi-infinite cavity. For the D-N cases in both the finite and the semi-infinite cavities, we find with a little surprise that a diverging flux emanates from the point where the BC changes but its property is completely different from that in the N-D case obtained in Ref. [16]. Furthermore, in the course that we reproduced the result of the N-D case, we found that such a diverging flux appears also in the N-D case in addition to the term of delta function squared but was overlooked in [16]. These results suggest that the divergence of flux, which would be a necessary condition for the quantum version of the cosmic censor to work, is not a special result in the N-D case. In addition, it is also suggested that the type of divergence sensitively depends on the combination of the initial and final BCs.

Here, let us give a few remarks on the analysis in this paper. The idealization of an instantaneous change of BCs, which is natural from the viewpoint of the formation of strong naked singularities, enables us to obtain all the results in analytic form. The particle creation by the rapid appearance and/or disappearance of a wall in a one-dimensional (1D) finite cavity was studied in Refs. [31-33]. In particular, the system with the instantaneous appearance and disappearance of a Dirichlet wall studied in [33] is more complex than but similar to the system in Sec. II of the present paper.

The organization of this paper is as follows. In Sec. II, we investigate the particle creation due to the instantaneous change of $\mathrm{BC}$ in a finite $1 \mathrm{D}$ cavity, for the N-D case (Sec. II B 1) and the D-N case (Sec. II B 2). The origin of the discrepancy between the result in Sec. II and Ref. [16] is clarified in Sec. III. In Sec. IV, the case of the semi-infinite cavity is analyzed. We conclude in Sec. V. The proof of consistency between different quantizations, called the unitarity relations, and some integration formulas are presented in Appendixes A and C, respectively. The result for the semi-infinite cavity in Sec. IV is reproduced in Appendix B with the Green-function method, which naturally involves the regularization of the vacuum expectation value of the energy-momentum tensor. We work in the natural units in which $c=\hbar=1$.

\section{FINITE CAVITY I}

\section{A. Quantization of massless scalar field}

We consider a free massless scalar field in a 1D cavity of which the length is $L$,

$\left(-\partial_{t}^{2}+\partial_{x}^{2}\right) \phi(t, x)=0, \quad-\infty<t<\infty, \quad 0<x<L$.

At the right boundary $x=L$, we assume the homogeneous Dirichlet boundary condition all the time,

$$
\phi(t, L)=0, \quad-\infty<t<\infty .
$$

At the left boundary $x=0$, we consider two kinds of boundary conditions. One is the homogeneous Neumann boundary condition, 


$$
\partial_{x} \phi(t, 0)=0 .
$$

Another is the Dirichlet boundary condition,

$$
\phi(t, 0)=0 .
$$

When boundary conditions (2) and (3) are imposed, a natural set of positive-energy mode functions $\left\{f_{n}\right\}$ is given by

$f_{n}(t, x)=\sqrt{\frac{2}{n \pi}} e^{-i p_{n} t} \cos \left(p_{n} x\right), \quad p_{n}:=\frac{n \pi}{2 L}, \quad n=1,3,5, \ldots$

In the rest of this paper, we suppose that $n$ and $n^{\prime}$ entirely denote odd natural numbers, otherwise denoted. The above mode functions satisfy the following orthonormal conditions:

$$
\left\langle f_{n}, f_{n^{\prime}}\right\rangle=-\left\langle f_{n}^{*}, f_{n^{\prime}}^{*}\right\rangle=\delta_{n n^{\prime}}, \quad\left\langle f_{n}, f_{n^{\prime}}^{*}\right\rangle=0,
$$

where the asterisk denotes the complex conjugate and $\langle$, denotes the Klein-Gordon inner product [21],

$$
\langle\phi, \psi\rangle:=i \int_{0}^{L}\left(\phi^{*} \partial_{t} \psi-\partial_{t} \phi^{*} \psi\right) d x .
$$

When boundary conditions (2) and (4) are imposed, a natural set of positive-energy mode functions $\left\{g_{m}\right\}$ is given by

$$
\begin{aligned}
g_{m}(t, x) & =\frac{1}{\sqrt{m \pi}} e^{-i q_{m} t} \sin \left(q_{m} x\right), \\
q_{m} & :=\frac{m \pi}{L}, \quad m=1,2,3, \ldots
\end{aligned}
$$

In the rest of this paper, we suppose that $m$ and $m^{\prime}$ entirely denote natural numbers, otherwise denoted. The above mode functions satisfy the following orthonormal conditions:

$\left\langle g_{m}, g_{m^{\prime}}\right\rangle=-\left\langle g_{m}^{*}, g_{m^{\prime}}^{*}\right\rangle=\delta_{m m^{\prime}}, \quad\left\langle g_{m}, g_{m^{\prime}}^{*}\right\rangle=0$.

Associated with the above two sets of mode functions, $\left\{f_{n}\right\}$ and $\left\{g_{m}\right\}$, there are two ways to quantize the scalar field. One is to expand the scalar field by $f_{n}$,

$$
\phi=\sum_{\substack{n=1 \\ n: \text { odd }}}^{\infty}\left(\boldsymbol{a}_{n} f_{n}+\boldsymbol{a}_{n}^{\dagger} f_{n}^{*}\right),
$$

and impose the commutation relations,

$$
\left[\boldsymbol{a}_{n}, \boldsymbol{a}_{n^{\prime}}^{\dagger}\right]=\delta_{n n^{\prime}}, \quad\left[\boldsymbol{a}_{n}, \boldsymbol{a}_{n^{\prime}}\right]=0 .
$$

By imposing the above commutation relations, the following equal-time canonical commutation relation is realized:

$$
\left[\boldsymbol{\phi}(t, x), \partial_{t} \phi\left(t, x^{\prime}\right)\right]=i \delta\left(x-x^{\prime}\right) .
$$

Then, $\boldsymbol{a}_{n}$ and $\boldsymbol{a}_{n}^{\dagger}$ are interpreted as the annihilation and creation operators, respectively. The vacuum state in which no particle corresponding to mode function $f_{n}$ exists is defined by

$\boldsymbol{a}_{n}\left|0_{f}\right\rangle=0, \quad n=1,3,5, \ldots, \quad\left\langle 0_{f} \mid 0_{f}\right\rangle=1$.

Another is to expand the field by $g_{m}$,

$$
\phi=\sum_{m=1}^{\infty}\left(\boldsymbol{b}_{m} g_{m}+\boldsymbol{b}_{m}^{\dagger} g_{m}^{*}\right),
$$

and impose the commutation relations,

$$
\left[\boldsymbol{b}_{m}, \boldsymbol{b}_{m^{\prime}}^{\dagger}\right]=\delta_{m m^{\prime}}, \quad\left[\boldsymbol{b}_{m}, \boldsymbol{b}_{m^{\prime}}\right]=0 .
$$

The vacuum state in which no particle corresponding to $g_{m}$ exists is defined by

$\boldsymbol{b}_{m}\left|0_{g}\right\rangle=0, \quad m=1,2,3, \ldots, \quad\left\langle 0_{g} \mid 0_{g}\right\rangle=1$.

Later, we will estimate the vacuum expectation value of the energy-momentum tensor for the scalar field. The energy-momentum tensor operator is written as $\boldsymbol{T}_{\mu \nu}=$ $\partial_{\mu} \phi \partial_{\nu} \phi-\frac{1}{2} \eta_{\mu \nu}(\partial \boldsymbol{\phi})^{2}$, where $\eta_{\mu \nu}=\operatorname{Diag}(-1,1)$ is the $(1+1)$-dimensional flat metric. Introducing double null coordinates, nonzero components of this tensor are

$$
\boldsymbol{T}_{ \pm \pm}=\left(\partial_{ \pm} \boldsymbol{\phi}\right)^{2}, \quad z_{ \pm}:=t \pm x .
$$

Note that the energy density and momentum density in the original Cartesian coordinates are $\boldsymbol{T}^{t t}=\boldsymbol{T}_{--}+\boldsymbol{T}_{++}$and $\boldsymbol{T}^{t x}=\boldsymbol{T}_{--}-\boldsymbol{T}_{++}$, respectively.

\section{B. Particle creation by instantaneous change of boundary condition}

Given the above quantization schemes, we investigate how the vacuum is excited when the boundary condition at the left boundary $x=0$ is instantaneously, say at $t=0$, changed from Neumann to Dirichlet (Sec. II B 1) and reversely (Sec. II B 2).

\section{From Neumann to Dirichlet}

First, we assume that the boundary condition at $x=0$ is Neumann (3) for $t<0$ and Dirichlet (4) for $t>0$, and that the quantum field is in vacuum $\left|0_{f}\right\rangle$ in the Heisenberg picture. See Fig. 1 for a schematic picture of this situation. Then, we investigate how the vacuum is excited due to the 


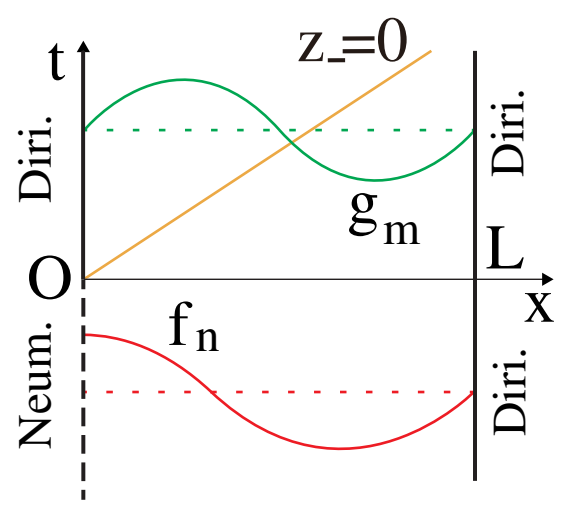

FIG. 1. The boundary condition at the left end of the domain $(x=0)$ instantaneously changes at $t=0$ from Neumann (dashed lines) to Dirichlet (solid lines). Spatial configurations of mode functions $f_{n}$ and $g_{m}$ are schematically depicted.

change of boundary condition by computing the spectrum and energy flux of created particles.

Let us expand $f_{n}$ by $g_{m}$,

$$
f_{n}=\sum_{m=1}^{\infty}\left(\alpha_{n m} g_{m}+\beta_{n m} g_{m}^{*}\right),
$$

where the expansion coefficients, called the Bogoliubov coefficients, are computed by

$$
\alpha_{n m}=\left\langle g_{m}, f_{n}\right\rangle, \quad \beta_{n m}=-\left\langle g_{m}^{*}, f_{n}\right\rangle .
$$

Using the explicit form of mode functions (5) and (8), we obtain

$\alpha_{n m}=\frac{2}{(2 m-n) \pi} \sqrt{\frac{2 m}{n}}, \quad \beta_{n m}=\frac{2}{(2 m+n) \pi} \sqrt{\frac{2 m}{n}}$.

Substituting Eq. (18) into Eq. (10), and comparing it with Eq. (14), we obtain

$$
\boldsymbol{b}_{m}=\sum_{\substack{n=1 \\ n \text { odd }}}^{\infty}\left(\alpha_{n m} \boldsymbol{a}_{n}+\beta_{n m}^{*} \boldsymbol{a}_{n}^{\dagger}\right) .
$$

Substituting Eq. (21) into Eq. (15) and using Eq. (11), we obtain

$$
\begin{aligned}
& \sum_{\substack{n=1 \\
n \text { :odd } \\
\infty}}^{\infty}\left(\alpha_{n m} \alpha_{n m^{\prime}}^{*}-\beta_{n m}^{*} \beta_{n m^{\prime}}\right)=\delta_{m m^{\prime}}, \\
& \sum_{\substack{n=1 \\
n \text { :odd }}}^{\infty}\left(\alpha_{n m} \beta_{n m^{\prime}}^{*}-\beta_{n m}^{*} \alpha_{n m^{\prime}}\right)=0,
\end{aligned}
$$

which should be satisfied for the two quantizations, Eqs. (10) and (14), to be consistent. In Appendix A 1, these consistency conditions, which we call unitarity relations, are shown to be satisfied by Bogoliubov coefficients (20).

The spectrum of created particles is given by the vacuum expectation value of number operator $\boldsymbol{b}_{m}^{\dagger} \boldsymbol{b}_{m}$,

$$
\left\langle 0_{f}\left|\boldsymbol{b}_{m}^{\dagger} \boldsymbol{b}_{m}\right| 0_{f}\right\rangle=\sum_{\substack{n=1 \\ n \text { :odd }}}^{\infty}\left|\beta_{n m}\right|^{2}=\frac{8}{\pi^{2}} \sum_{\substack{n=1 \\ n \text { :odd }}}^{\infty} \frac{m}{n(n+2 m)^{2}} .
$$

Note that this is finite but its summation over $m$, the total number of created particles, is divergent. This implies that the Fock-space representation associated with $\boldsymbol{a}_{n}$ is unitarily inequivalent to that associated with $\boldsymbol{b}_{m}$ [22].

The vacuum expectation value of the energy-momentum tensor before the change of boundary condition at $t=0$ is computed by substituting Eq. (10) into Eq. (17), and using Eqs. (11), (13), and (5) as

$$
\left\langle 0_{f}\left|\boldsymbol{T}_{ \pm \pm}\right| 0_{f}\right\rangle_{t<0}=\sum_{\substack{n=1 \\ n: \text { odd }}}^{\infty}\left|\partial_{ \pm} f_{n}\right|^{2}=\frac{\pi}{8 L^{2}} \sum_{\substack{n=1 \\ n \text { :odd }}}^{\infty} n .
$$

This represents the Casimir energy density [1], which can be made finite with standard regularization schemes [21].

The most interesting quantity is the vacuum expectation value of the energy-momentum tensor after $t=0$. Substituting Eq. (14) into Eq. (17) and using Eq. (21), we obtain

$$
\begin{aligned}
& \left\langle 0_{f}\left|\boldsymbol{T}_{ \pm \pm}\right| 0_{f}\right\rangle_{t>0} \\
& =\sum_{\substack{n=1 \\
n: \text { odd }}}^{\infty} \sum_{m=1}^{\infty} \sum_{m^{\prime}=1}^{\infty}\left[\left(\alpha_{n m} \beta_{n m^{\prime}}+\alpha_{n m^{\prime}} \beta_{n m}\right) \operatorname{Re}\left(\partial_{ \pm} g_{m} \partial_{ \pm} g_{m^{\prime}}\right)\right. \\
& \left.\quad+\left(\alpha_{n m} \alpha_{n m^{\prime}}+\beta_{n m} \beta_{n m^{\prime}}\right) \operatorname{Re}\left(\partial_{ \pm} g_{m} \partial_{ \pm} g_{m^{\prime}}^{*}\right)\right]
\end{aligned}
$$

To derive Eq. (25), we symmetrize it with respect to dummy indices $m$ and $m^{\prime}$, and use the fact that $\alpha_{n m}$ and $\beta_{n m}$ are real. Using the explicit expressions of Bogoliubov coefficients (20) and mode function (8), we obtain

$$
\begin{aligned}
& \left\langle 0_{f}\left|\boldsymbol{T}_{ \pm \pm}\right| 0_{f}\right\rangle_{t>0} \\
& =\frac{1}{2 \pi L^{2}} \sum_{\substack{n=1 \\
n \text { odd }}}^{\infty}\left(\frac{1}{4 n}\left[4 \sum_{m=1}^{\infty} \cos \left(q_{m} z_{ \pm}\right)+n^{2} \sum_{m=1}^{\infty} \frac{\cos \left(q_{m} z_{ \pm}\right)}{m^{2}-(n / 2)^{2}}\right]^{2}\right. \\
& \left.\quad+n\left[\sum_{m=1}^{\infty} \frac{m \sin \left(q_{m} z_{ \pm}\right)}{m^{2}-(n / 2)^{2}}\right]^{2}\right)
\end{aligned}
$$

This is an even function of $z_{ \pm}$with period $2 L$ since it is invariant under reflection $z_{ \pm} \rightarrow-z_{ \pm}$and translation $z_{ \pm} \rightarrow z_{ \pm}+2 L$. Therefore, it is sufficient to calculate it in $0 \leq z_{ \pm}<2 L$, and then generalize the obtained expression appropriately to one valid in the entire domain. 
The first and second summations over $m$ in Eq. (26) can be computed to give

$$
\begin{aligned}
\left\langle 0_{f}\left|\boldsymbol{T}_{ \pm \pm}\right| 0_{f}\right\rangle_{t>0} & \\
= & \frac{1}{2 \pi L^{2}} \sum_{\substack{n=1 \\
n \text { :odd }}}^{\infty}\left(\frac { 1 } { 4 n } \left[16 L^{2} \delta^{2}\left(z_{ \pm}\right)\right.\right. \\
& \left.\left.\quad+n^{2} \pi^{2} \sin ^{2}\left(p_{n} z_{ \pm}\right)\right]+n\left[\sum_{m=1}^{\infty} \frac{m \sin \left(q_{m} z_{ \pm}\right)}{m^{2}-(n / 2)^{2}}\right]^{2}\right),
\end{aligned}
$$

which is valid in $0 \leq z_{ \pm}<2 L$, using the following formulas:

$$
\begin{aligned}
& \sum_{k=1}^{\infty} \cos \left(\frac{2 k \pi}{a} y\right)=-\frac{1}{2}+\frac{a}{2} \sum_{\ell=-\infty}^{\infty} \delta(y-\ell a), \\
&(-\infty<y<\infty), \\
& \sum_{k=1}^{\infty} \frac{\cos k y}{k^{2}-a^{2}}=-\frac{\pi}{2 a} \cos [a(\pi-y)] \operatorname{cosec}(a \pi)+\frac{1}{2 a^{2}}, \\
&(0 \leq y \leq 2 \pi) .
\end{aligned}
$$

See Ref. [34] [p. 730] for the second formula.

For $z_{ \pm}=0$, from Eq. (27), we have

$$
\left\langle 0_{f}\left|\boldsymbol{T}_{ \pm \pm}\right| 0_{f}\right\rangle_{t>0}=\frac{2}{\pi} \sum_{\substack{n=1 \\ n \text { :odd }}}^{\infty} \frac{\delta^{2}(0)}{n} \quad\left(z_{ \pm}=0\right) .
$$

For $0<z_{ \pm}<2 L$, the rest summation over $m$ in Eq. (27) can be computed to give

$$
\left\langle 0_{f}\left|\boldsymbol{T}_{ \pm \pm}\right| 0_{f}\right\rangle_{t>0}=\frac{\pi}{8 L^{2}} \sum_{\substack{n=1 \\ n \text { :odd }}}^{\infty} n \quad\left(0<z_{ \pm}<2 L\right),
$$

using the following formula [34] [p. 730]:

$$
\sum_{k=1}^{\infty} \frac{k \sin k y}{k^{2}-a^{2}}=\frac{\pi}{2} \sin [a(\pi-y)] \operatorname{cosec}(a \pi) \quad(0<y<2 \pi) .
$$

Combining Eqs. (30) and (31), we obtain

$$
\begin{aligned}
\left\langle 0_{f}\left|\boldsymbol{T}_{ \pm \pm}\right| 0_{f}\right\rangle_{t>0}= & \frac{2}{\pi} \sum_{\substack{n=1 \\
n \text { :odd }}}^{\infty} \frac{\delta^{2}\left(z_{ \pm}\right)}{n} \\
& +\left\{\begin{array}{ll}
0 & \left(z_{ \pm}=0\right) \\
\frac{\pi}{8 L^{2}} \sum_{\substack{n=1 \\
n: \text { odd }}}^{\infty} n & \left(0<z_{ \pm}<2 L\right)
\end{array} .\right.
\end{aligned}
$$

This is the expression for $0 \leq z_{ \pm}<2 L$, which we wanted to know. Extending the domain of Eq. (33), we obtain
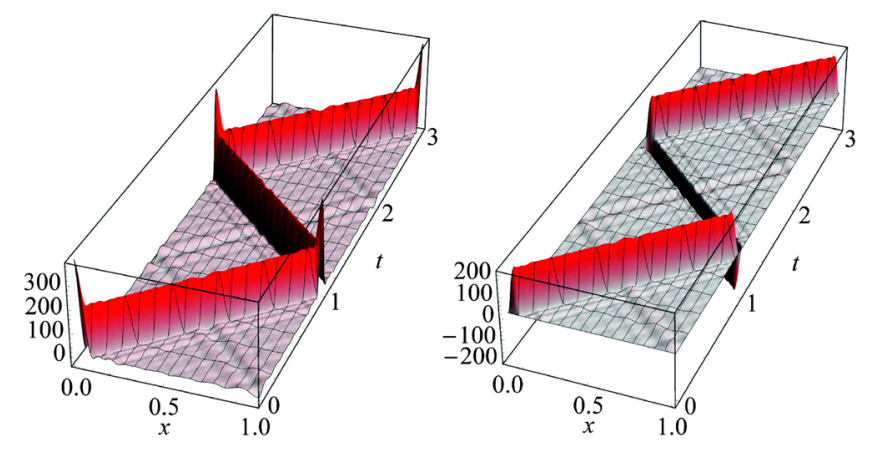

FIG. 2. Vacuum expectation values of energy density $\left\langle 0_{f}\left|\left(\boldsymbol{T}_{--}+\boldsymbol{T}_{++}\right)\right| 0_{f}\right\rangle_{t>0}$ (left) and momentum density $\left\langle 0_{f}\right|\left(\boldsymbol{T}_{--}-\right.$ $\left.\boldsymbol{T}_{++}\right)\left|0_{f}\right\rangle_{t>0}$ (right) with cutoff, from which the uniform Casimir contribution is subtracted. We set $L=1$, and the summation over modes in Eq. (26) is taken up to $n=m=13$. The exact results without cutoff are given by Eq. (34).

$$
\begin{aligned}
\left\langle 0_{f}\left|\boldsymbol{T}_{ \pm \pm}\right| 0_{f}\right\rangle_{t>0}= & \frac{2}{\pi} \sum_{\substack{n=1 \\
n: \text { odd }}}^{\infty} \frac{1}{n} \sum_{\ell=-\infty}^{\infty} \delta^{2}\left(z_{ \pm}-2 \ell L\right) \\
& + \begin{cases}0 & \left(z_{ \pm}=2 \ell L, \ell \in \boldsymbol{Z}\right) \\
\frac{\pi}{8 L^{2}} \sum_{\substack{n=1 \\
n \text { odd }}}^{\infty} n & \text { (otherwise) }\end{cases}
\end{aligned}
$$

Let us consider the meaning of two terms in Eq. (34). The first term, the delta function squared multiplied by the logarithmically divergent series, represents the diverging flux emanating from the origin $(t, x)=(0,0)$ and localizing on the null lines (Fig. 2). The dependence of energy density on the delta function squared implies also the divergence of total energy emitted. This component of flux is similar to that predicted in the topology change of the 1D universe [18] and is the same as that predicted in the formation of a strong naked singularity [16].

The second term, at first glance, seems to represent the ambient Casimir energy just as Eq. (24), which is negative and finite after a regularization, and its vanishing on the null lines. As will be explicitly shown in the semi-infinite cavity case (see Sec. IV and Appendix B), however, this is not the case. The second term represents the divergence on the null lines after an appropriate regularization in fact. A simple understanding of such an appearance of divergence is possible as follows. A regularization corresponds to the subtraction of the spatially uniform diverging energy density due to the zero-point oscillation. Therefore, if one subtracts such a uniform diverging quantity from Eq. (34), leading to the regularization of the ambient Casimir term, a divergence appears on the null lines $z_{ \pm}=2 \ell L(\ell \in Z)$.

As far as the present author knows, the second kind of diverging flux was first found in the particle creation due to the instantaneous appearance of the Dirichlet wall in a cavity [33]. It was confirmed in the same paper that such a divergence appears in the instantaneous limit of smooth formation of a Dirichlet wall in the cavity analyzed in [32]. 
It is suspicious that the second kind of flux component does not appear in the analysis of Ishibashi and Hosoya [16], since their system is quite similar to the present one. Thus, we will revisit their analysis in Sec. III and find that the component was overlooked in [16].

\section{From Dirichlet to Neumann}

We assume that the boundary condition at $x=0$ is Dirichlet (4) for $t<0$ and Neumann (3) for $t>0$, and that the quantum field is in vacuum $\left|0_{g}\right\rangle$. See Fig. 3 for a schematic picture of the situation. Since this situation is a kind of time reversal of that in Sec. II B 1, most parts of the calculation can be reused but the results are different.

Let us expand $g_{m}$ by $f_{n}$,

$$
g_{m}=\sum_{\substack{n=1 \\ n \text { odd }}}^{\infty}\left(\rho_{m n} f_{n}+\sigma_{m n} f_{n}^{*}\right),
$$

where the expansion coefficients are given by

$$
\rho_{m n}=\left\langle f_{n}, g_{m}\right\rangle=\alpha_{n m}^{*}, \quad \sigma_{m n}=-\left\langle f_{n}^{*}, g_{m}\right\rangle=-\beta_{n m} .
$$

Here, $\alpha_{n m}$ and $\beta_{n m}$ are given by Eq. (20).

Substituting Eq. (35) into Eq. (14), and comparing it with Eq. (10), we obtain

$$
\boldsymbol{a}_{n}=\sum_{m=1}^{\infty}\left(\rho_{m n} \boldsymbol{b}_{m}+\sigma_{m n}^{*} \boldsymbol{b}_{m}^{\dagger}\right)
$$

Substituting Eq. (37) into Eq. (11), and using Eq. (15), we obtain

$$
\begin{aligned}
& \sum_{m=1}^{\infty}\left(\rho_{m n} \rho_{m n^{\prime}}^{*}-\sigma_{m n}^{*} \sigma_{m n^{\prime}}\right)=\delta_{n n^{\prime}}, \\
& \sum_{m=1}^{\infty}\left(\rho_{m n} \sigma_{m n^{\prime}}^{*}-\sigma_{m n}^{*} \rho_{m n^{\prime}}\right)=0,
\end{aligned}
$$

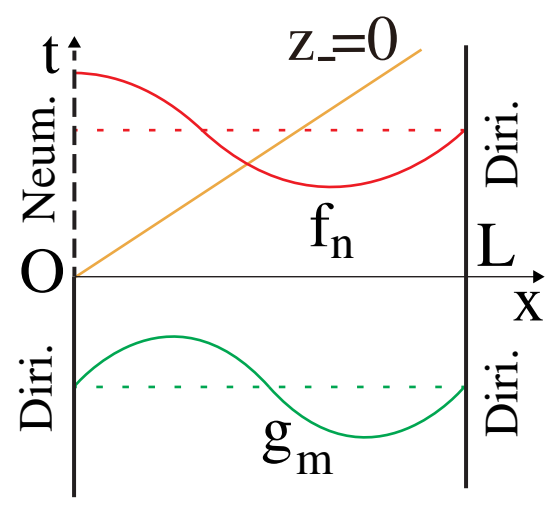

FIG. 3. The boundary condition at the left end of the domain $(x=0)$ instantaneously changes at $t=0$ from Dirichlet (solid lines) to Neumann (dashed lines). Spatial configurations of mode functions $g_{m}$ and $f_{n}$ are schematically depicted. which should be satisfied again for the two quantizations, Eqs. (10) and (14), to be consistent. It is shown in Appendix A 2 that the Bogoliubov coefficients given by Eq. (36) indeed satisfy unitarity relations (38).

The vacuum expectation value of number operator $\boldsymbol{a}_{n}^{\dagger} \boldsymbol{a}_{n}$, representing the energy spectrum of created particles, is computed as

$$
\left\langle 0_{g}\left|\boldsymbol{a}_{n}^{\dagger} \boldsymbol{a}_{n}\right| 0_{g}\right\rangle=\sum_{m=1}^{\infty}\left|\sigma_{m n}\right|^{2}=\frac{8}{\pi^{2}} \sum_{m=1}^{\infty} \frac{m}{n(n+2 m)^{2}} .
$$

This and its summation over odd $n$, i.e., the total number of created particles, are divergent. This implies that the Fockspace representation associated with $\boldsymbol{b}_{m}$ is unitarily inequivalent to that associated with $\boldsymbol{a}_{n}$ [22].

The vacuum expectation value of the energy-momentum tensor before the change of boundary condition at $t=0$ is computed by substituting Eq. (14) into Eq. (17), and using the explicit expression of mode function (8),

$$
\left\langle 0_{g}\left|\boldsymbol{T}_{ \pm \pm}\right| 0_{g}\right\rangle_{t<0}=\sum_{m=1}^{\infty}\left|\partial_{ \pm} g_{m}\right|^{2}=\frac{\pi}{4 L^{2}} \sum_{m=1}^{\infty} m
$$

This represents the Casimir energy density, which can be made finite by standard renormalization procedures [21].

The vacuum expectation value of the energy-momentum tensor after $t=0$ is computed by substituting Eq. (10) into Eq. (17), and using Eq. (37), as

$$
\begin{aligned}
& \left\langle 0_{g}\left|\boldsymbol{T}_{ \pm \pm}\right| 0_{g}\right\rangle_{t>0} \\
& =\sum_{m=1}^{\infty} \sum_{\substack{n=1 \\
n: \text { odd } n_{n^{\prime}}:=1}}^{\infty} \sum_{m=1}^{\infty}\left[\left(\rho_{m n} \sigma_{m n^{\prime}}+\rho_{m n^{\prime}} \sigma_{m n}\right) \operatorname{Re}\left(\partial_{ \pm} f_{n} \partial_{ \pm} f_{n^{\prime}}\right)\right. \\
& \left.\quad+\left(\rho_{m n} \rho_{m n^{\prime}}+\sigma_{m n} \sigma_{m n^{\prime}}\right) \operatorname{Re}\left(\partial_{ \pm} f_{n} \partial_{ \pm} f_{n^{\prime}}^{*}\right)\right]
\end{aligned}
$$

which we symmetrize with respect to dummy indices $n$ and $n^{\prime}$, and we use the fact that $\rho_{m n}$ and $\sigma_{m n}$ are real.

Using the explicit form of Bogoliubov coefficients and mode functions, Eqs. (36), (20), and (5), we obtain

$$
\begin{aligned}
\left\langle 0_{g}\left|\boldsymbol{T}_{ \pm \pm}\right| 0_{g}\right\rangle_{t>0}= & \frac{4}{\pi L^{2}} \sum_{m=1}^{\infty}\left(4 m^{3}\left[\sum_{\substack{n=1 \\
n: \text { odd }}}^{\infty} \frac{\cos \left(p_{n} z_{ \pm}\right)}{n^{2}-(2 m)^{2}}\right]^{2}\right. \\
& \left.+m\left[\sum_{\substack{n=1 \\
n \text { :odd }}}^{\infty} \frac{n \sin \left(p_{n} z_{ \pm}\right)}{n^{2}-(2 m)^{2}}\right]^{2}\right) .
\end{aligned}
$$

This is an even function of $z_{ \pm}$with period $2 L$, since it is invariant under reflection $z_{ \pm} \rightarrow-z_{ \pm}$and translation $z_{ \pm} \rightarrow z_{ \pm}+2 L$. Therefore, it is sufficient to calculate it in $0 \leq z_{ \pm}<2 L$ and generalize it appropriately to one valid in the entire domain.

The first summation over odd $n$ in Eq. (42) can be computed to give 


$$
\begin{aligned}
& \left\langle 0_{g}\left|\boldsymbol{T}_{ \pm \pm}\right| 0_{g}\right\rangle_{t>0} \\
& =\frac{4}{\pi L^{2}} \sum_{m=1}^{\infty}\left(\frac{m \pi^{2}}{16} \sin ^{2}\left(q_{m} z_{ \pm}\right)+m\left[\sum_{\substack{n=1 \\
n: \text { odd }}}^{\infty} \frac{n \sin \left(p_{n} z_{ \pm}\right)}{n^{2}-(2 m)^{2}}\right]^{2}\right),
\end{aligned}
$$

which is valid in $0 \leq z_{ \pm}<2 L$. Here, we have used the following formula [34] [p. 733]:

$$
\begin{aligned}
\sum_{k=0}^{\infty} \frac{\cos [(2 k+1) y]}{(2 k+1)^{2}-a^{2}}= & \frac{\pi}{4 a} \sin \left[\frac{a}{2}(\pi-2 y)\right] \sec \left(\frac{a \pi}{2}\right) \\
& (0 \leq y \leq \pi) .
\end{aligned}
$$

It is noted here that there are typos in Ref. [34] [p. 733] about formulas (44) and (47) (see below).

For $z_{ \pm}=0$, from Eq. (43), we have

$$
\left\langle 0_{g}\left|\boldsymbol{T}_{ \pm \pm}\right| 0_{g}\right\rangle_{t>0}=0 \quad\left(z_{ \pm}=0\right) .
$$

For $0<z_{ \pm}<2 L$, the rest summation over odd $n$ in Eq. (43) can be computed to give

$$
\left\langle 0_{g}\left|\boldsymbol{T}_{ \pm \pm}\right| 0_{g}\right\rangle_{t>0}=\frac{\pi}{4 L^{2}} \sum_{m=1}^{\infty} m \quad\left(0<z_{ \pm}<2 L\right),
$$

using the following formula [34] [p. 733]:

$$
\begin{aligned}
\sum_{k=0}^{\infty} \frac{(2 k+1) \sin [(2 k+1) y]}{(2 k+1)^{2}-a^{2}}= & \frac{\pi}{4} \cos \left[\frac{a}{2}(\pi-2 y)\right] \sec \left(\frac{a \pi}{2}\right) \\
& (0<y<\pi) .
\end{aligned}
$$

Combining Eqs. (45) and (46), and extending the domain periodically into the entire domain, we have

$$
\left\langle 0_{g}\left|\boldsymbol{T}_{ \pm \pm}\right| 0_{g}\right\rangle_{t>0}=\left\{\begin{array}{ll}
0 & \left(z_{ \pm}=2 \ell L, \ell \in \boldsymbol{Z}\right) \\
\frac{\pi}{4 L^{2}} \sum_{m=1}^{\infty} m & \text { (otherwise) }
\end{array} .\right.
$$

Comparing the above result with that in the N-D case (34), one sees that there is no flux component of the delta function squared in this case. As will be explicitly shown in the semi-infinite cavity case (Sec. IV and Appendix B), Eq. (48) represents the nonrenormalizable diverging flux localized on the null lines $z_{ \pm}=2 \ell L(\ell \in Z)$ and the ambient Casimir energy. Thus, the diverging flux emanates from origin $(t, x)=(0,0)$ and propagates along the null lines in a way similar to Fig. 2.

\section{FINITE CAVITY II: REVISIT ISHIBASHI-HOSOYA [16]}

As seen in Sec. II, the vacuum expectation value of the energy-momentum tensor has two components in the N-D case as Eq. (34) and one component in the D-N case as Eq. (48). The origin of such a difference between the N-D and D-N cases will be discussed in the Conclusion. Here, let us look into the consistency between these results and a relevant past work.

In Ref. [16], the authors considered the instantaneous change of boundary condition at both sides of the finite cavity. The boundary conditions for $t<0$ are Neumann at both sides and those for $t>0$ are Dirichlet at both sides, which we call the NN-DD case. Since this NN-DD case resembles the N-D case, one can expect similar results. Namely, we expect that two diverging flux components appear also in the NN-DD case. Reference [16], however, concludes the flux involves only the component of the delta function squared. Therefore, we will reconsider here the system adopted in [16], and we find that the other component was overlooked.

\section{A. Quantization of massless scalar field}

We consider the situation that the Neumann boundary condition is imposed at $x=0$ and $x=L$ for $t<0$, while the Dirichlet boundary condition is imposed at $x=0$ and $x=L$ for $t>0$ (see Fig. 4).

In this case, a normalized positive-energy mode function for $t<0$ is given by

$h_{k}(t, x)=\frac{1}{\sqrt{k \pi}} e^{-i r_{k} t} \cos \left(r_{k} x\right), \quad r_{k}:=\frac{k \pi}{L}, \quad k=1,2,3, \ldots$

A normalized mode function for $t>0$ is given by Eq. (8).

The scalar field is quantized by expanding it by a set of mode functions $\left\{h_{k}\right\}$ and an additional zero-mode function $\boldsymbol{h}_{0}$, being spatially uniform, as

$$
\boldsymbol{\phi}=\boldsymbol{h}_{0}+\sum_{k=1}^{\infty}\left(\boldsymbol{c}_{k} h_{k}+\boldsymbol{c}_{k}^{\dagger} h_{k}^{*}\right), \quad \boldsymbol{h}_{0}=\frac{1}{\sqrt{L}}(\boldsymbol{Q}+t \boldsymbol{P}) .
$$

Here, $\boldsymbol{Q}$ and $\boldsymbol{P}$ are Hermitian $\left(\boldsymbol{Q}^{\dagger}=\boldsymbol{Q}, \boldsymbol{P}^{\dagger}=\boldsymbol{P}\right)$, and the following commutation relations are imposed:

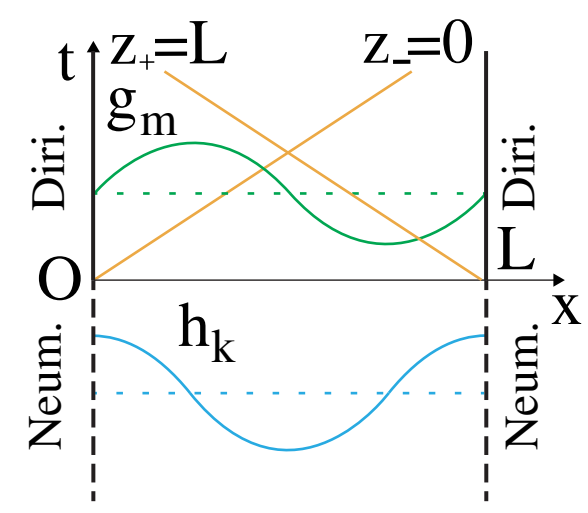

FIG. 4. The boundary conditions at $x=0$ and $x=L$ are instantaneously changed at $t=0$ from Neumann (dashed lines) to Dirichlet (solid lines). Spatial configurations of mode functions $h_{k}$ and $g_{m}$ are schematically depicted. 


$$
\begin{gathered}
{[\boldsymbol{Q}, \boldsymbol{P}]=i, \quad\left[\boldsymbol{Q}, \boldsymbol{c}_{k}\right]=\left[\boldsymbol{P}, \boldsymbol{c}_{k}\right]=0,} \\
{\left[\boldsymbol{c}_{k}, \boldsymbol{c}_{k^{\prime}}^{\dagger}\right]=\delta_{k k^{\prime}}, \quad\left[\boldsymbol{c}_{k}, \boldsymbol{c}_{k^{\prime}}\right]=0 .}
\end{gathered}
$$

Note that zero-mode $\boldsymbol{h}_{0}$, which exists because the boundary conditions are Neumann at both ends, is indispensable to realize the equal-time commutation relation (12) using commutation relations (51).

\section{B. Particle creation by instantaneous change of boundary condition: From Neumann-Neumann to Dirichlet-Dirichlet}

Let us expand $\boldsymbol{h}_{0}$ and $h_{k}$ by $g_{m}$,

$$
\boldsymbol{h}_{0}=\sum_{m=1}^{\infty}\left(\boldsymbol{\xi}_{m} g_{m}+\boldsymbol{\xi}_{m}^{\dagger} g_{m}^{*}\right), \quad h_{k}=\sum_{m=1}^{\infty}\left(\xi_{k m} g_{m}+\zeta_{k m} g_{m}^{*}\right),
$$

where the Bogoliubov coefficients are given by

$$
\boldsymbol{\xi}_{m}=\left\langle g_{m}, \boldsymbol{h}_{0}\right\rangle, \quad \boldsymbol{\xi}_{k m}=\left\langle g_{m}, h_{k}\right\rangle, \quad \zeta_{k m}=-\left\langle g_{m}^{*}, h_{k}\right\rangle .
$$

Using the explicit form of mode functions (8) and (49), and Eq. (50), Bogoliubov coefficients (53) are computed as

$$
\begin{aligned}
\boldsymbol{\xi}_{m} & =\frac{2}{\sqrt{m \pi L}}\left(\boldsymbol{Q}+i \frac{L}{m \pi} \boldsymbol{P}\right) \delta_{m: \text { odd }}, \\
\xi_{k m} & =-\frac{2}{(k-m) \pi} \sqrt{\frac{m}{k}} \delta_{k+m: \text { odd }}, \\
\zeta_{k m} & =\frac{2}{(k+m) \pi} \sqrt{\frac{m}{k}} \delta_{k+m: \text { odd }} .
\end{aligned}
$$

Here, we have introduced the following symbols:

$\delta_{k: \text { odd }}:=\frac{1-(-1)^{k}}{2}, \quad \delta_{k: \text { even }}:=\frac{1+(-1)^{k}}{2}, \quad k \in \boldsymbol{Z}$.

Substituting Eq. (52) into Eq. (50), and comparing it with Eq. (14), we have

$$
\boldsymbol{b}_{m}=\boldsymbol{\xi}_{m}+\sum_{k=1}^{\infty}\left(\xi_{k m} \boldsymbol{c}_{k}+\zeta_{k m}^{*} \boldsymbol{c}_{k}^{\dagger}\right)
$$

Substituting Eq. (57) into Eq. (15) and using Eq. (51), we obtain the unitarity relations

$$
\begin{aligned}
& {\left[\boldsymbol{\xi}_{m}, \boldsymbol{\xi}_{m^{\prime}}^{\dagger}\right]+\sum_{k=1}^{\infty}\left(\xi_{k m} \xi_{k m^{\prime}}^{*}-\zeta_{k m}^{*} \zeta_{k m^{\prime}}\right)=\delta_{m m^{\prime}}} \\
& {\left[\boldsymbol{\xi}_{m}, \boldsymbol{\xi}_{m^{\prime}}\right]+\sum_{k=1}^{\infty}\left(\xi_{k m} \zeta_{k m^{\prime}}^{*}-\zeta_{k m}^{*} \xi_{k m^{\prime}}\right)=0}
\end{aligned}
$$

In Appendix A 3, we will show that the operators given in Eqs. (54) and (55) satisfy unitarity relations (58).

We define the vacuum in which no particle corresponding to $\boldsymbol{h}_{0}$ or $h_{k}$ exists,

$$
\boldsymbol{P}\left|0_{h}\right\rangle=\boldsymbol{c}_{k}\left|0_{h}\right\rangle=0, \quad k=1,2,3, \ldots
$$

Then, the spectrum of created particles are given by the expectation value of number operator $\boldsymbol{b}_{m}^{\dagger} \boldsymbol{b}_{m}$,

$$
\begin{aligned}
\left\langle 0_{h}\left|\boldsymbol{b}_{m}^{\dagger} \boldsymbol{b}_{m}\right| 0_{h}\right\rangle= & \left\langle 0_{h}\left|\boldsymbol{\xi}_{m}^{\dagger} \boldsymbol{\xi}_{m}\right| 0_{h}\right\rangle+\sum_{k=1}^{\infty}\left|\zeta_{k m}\right|^{2} \\
= & \frac{4}{m^{2} \pi^{2}}\left(\frac{m \pi}{L}\left\langle 0_{h}\left|\boldsymbol{Q}^{2}\right| 0_{h}\right\rangle-1\right) \delta_{m: \text { odd }} \\
& +\frac{4}{\pi^{2}} \sum_{k=1}^{\infty} \frac{m}{k(k+m)^{2}} \delta_{k+m \text { :odd }} .
\end{aligned}
$$

The vacuum expectation value of the energy-momentum tensor before the change of boundary conditions at $t=0$ is computed by substituting Eq. (50) into Eq. (17), and using an explicit form of mode function (49) as

$$
\left\langle 0_{h}\left|\boldsymbol{T}_{ \pm \pm}\right| 0_{h}\right\rangle_{t<0}=\sum_{k=1}^{\infty}\left|\partial_{ \pm} h_{k}\right|^{2}=\frac{\pi}{4 L^{2}} \sum_{k=1}^{\infty} k .
$$

This represents the Casimir energy density, which can be made finite by standard regularization schemes such as the $\zeta$-function regularization, the point-splitting regularization, and so on [21].

The vacuum expectation value of the energy-momentum tensor after $t=0$ is computed by substituting Eq. (14) into Eq. (17), and using Eq. (57),

$$
\begin{aligned}
\left\langle 0_{h}\left|\boldsymbol{T}_{ \pm \pm}\right| 0_{h}\right\rangle_{t>0}= & \sum_{\substack{m=1 \\
m: \text { odd } \\
m^{\prime}: \text { :odd }}}^{\infty} \sum^{\infty}\left[\frac{8\left\langle 0_{h}\left|\boldsymbol{Q}^{2}\right| 0_{h}\right\rangle}{\pi L \sqrt{m m^{\prime}}} \operatorname{Re}\left(\partial_{ \pm} g_{m} \partial_{ \pm} g_{m^{\prime}}+\partial_{ \pm} g_{m} \partial_{ \pm} g_{m^{\prime}}^{*}\right)\right. \\
& \left.+\frac{4 i}{\sqrt{\pi^{2} m^{3} m^{\prime 3}}} \operatorname{Im}\left[\left(m+m^{\prime}\right) \partial_{ \pm} g_{m} \partial_{ \pm} g_{m^{\prime}}-\left(m-m^{\prime}\right) \partial_{ \pm} g_{m} \partial_{ \pm} g_{m^{\prime}}^{*}\right]\right] \\
& +\sum_{k=1}^{\infty} \sum_{m=1}^{\infty} \sum_{m^{\prime}=1}^{\infty}\left[\left(\xi_{k m} \zeta_{k m^{\prime}}+\zeta_{k m} \xi_{k m^{\prime}}\right) \operatorname{Re}\left(\partial_{ \pm} g_{m} \partial_{ \pm} g_{m^{\prime}}\right)+\left(\xi_{k m} \xi_{k m^{\prime}}+\zeta_{k m} \zeta_{k m^{\prime}}\right) \operatorname{Re}\left(\partial_{ \pm} g_{m} \partial_{ \pm} g_{m^{\prime}}^{*}\right)\right]
\end{aligned}
$$

which we symmetrize with respect to dummy indices $m$ and $m^{\prime}$, and we have used the fact that $\xi_{k m}$ and $\zeta_{k m}$ are real. 
Using an explicit form of mode functions (8) and Bogoliubov coefficients (55), we obtain

$$
\begin{aligned}
\left\langle 0_{h}\left|\boldsymbol{T}_{ \pm \pm}\right| 0_{h}\right\rangle_{t>0}= & \frac{4\left\langle 0_{h}\left|\boldsymbol{Q}^{2}\right| 0_{h}\right\rangle}{L^{3}}\left[\sum_{\substack{m=1 \\
m: \text { odd }}}^{\infty} \cos \left(q_{m} z_{ \pm}\right)\right]^{2}-\frac{4 i}{\pi L^{2}} \sum_{\substack{m=1 \\
m \text { :odd }}}^{\infty} \frac{\sin \left(q_{m} z_{ \pm}\right)}{m} \sum_{\substack{m=1 \\
m \text { :odd }}}^{\infty} \cos \left(q_{m} z_{ \pm}\right) \\
& +\frac{4}{\pi L^{2}} \sum_{\substack{k=1 \\
k: \text { odd }}}^{\infty}\left(\frac{1}{k}\left[\sum_{\substack{m=2 \\
m}}^{\infty} \cos \left(q_{m} z_{ \pm}\right)+k^{2} \sum_{\substack{m=2 \\
m \text { :even }}}^{\infty} \frac{\cos \left(q_{m} z_{ \pm}\right)}{m^{2}-k^{2}}\right]^{2}+k\left[\sum_{\substack{m=2 \\
m \text { :even }}}^{\infty} \frac{m \sin \left(q_{m} z_{ \pm}\right)}{m^{2}-k^{2}}\right]^{2}\right) \\
& +\frac{4}{\pi L^{2}} \sum_{\substack{k=2 \\
k: \text { :ven }}}^{\infty}\left(\frac{1}{k}\left[\sum_{\substack{m=1 \\
m: \text { odd }}}^{\infty} \cos \left(q_{m} z_{ \pm}\right)+k^{2} \sum_{\substack{m=1 \\
m: \text { odd }}}^{\infty} \frac{\cos \left(q_{m} z_{ \pm}\right)}{m^{2}-k^{2}}\right]^{2}+k\left[\sum_{\substack{m=1 \\
m \text { :odd }}}^{\infty} \frac{m \sin \left(q_{m} z_{ \pm}\right)}{m^{2}-k^{2}}\right]^{2}\right) .
\end{aligned}
$$

The summations over odd $m$ in the first two terms of Eq. (63), both of which are the contributions of the zero mode, are computed using the following formulas:

$$
\begin{gathered}
\sum_{\substack{k=1 \\
k \text { odd }}}^{\infty} \frac{1}{k} \sin \left(\frac{2 k \pi}{a} y\right)=\frac{\pi}{4} \sum_{\ell=-\infty}^{\infty}(-1)^{\ell} \Pi_{0}^{a / 2}\left(y-\frac{a}{2} \ell\right) \quad(-\infty<y<\infty), \\
\sum_{\substack{k=1 \\
k: \text { odd }}}^{\infty} \cos \left(\frac{2 k \pi}{a} y\right)=\frac{a}{4} \sum_{\ell=-\infty}^{\infty}(-1)^{\ell} \delta\left(y-\frac{a}{2} \ell\right) \quad(-\infty<y<\infty),
\end{gathered}
$$

where $\Pi_{a}^{b}(x)$ is the rectangular function defined as

$$
\Pi_{a}^{b}(x):=\int_{a}^{b} \delta(x-y) d y=\left\{\begin{array}{ll}
0 & (x<a, b<x) \\
\frac{1}{2} & (x=a, b) \\
1 & (a<x<b)
\end{array} .\right.
$$

The rest summations over odd and even $m$ in Eq. (63) are computed using formulas (28), (29), (32), (44), and (47) in addition to the above formulas, to obtain

$$
\left\langle 0_{h}\left|\boldsymbol{T}_{ \pm \pm}\right| 0_{h}\right\rangle_{t>0}=\left(\frac{\left\langle 0_{h}\left|\boldsymbol{Q}^{2}\right| 0_{h}\right\rangle}{L}+\frac{1}{\pi} \sum_{k=1}^{\infty} \frac{1}{k}\right) \sum_{\ell=-\infty}^{\infty} \delta^{2}\left(z_{ \pm}-\ell L\right)+\left\{\begin{array}{ll}
0 & \left(z_{ \pm}=\ell L, \ell \in \boldsymbol{Z}\right) \\
\frac{\pi}{4 L^{2}} \sum_{k=1}^{\infty} k & \text { (otherwise) }
\end{array} .\right.
$$

After setting $L=\pi$ and regularizing the diverging summation as $\sum_{k=1}^{\infty} k=-\frac{1}{12}$ by the $\zeta$-function regularization, Eq. (67) should be equal to Eq. (31) of Ref. [16]. The vanishing of Casimir energy on the null lines in Eq. (67), however, has no counterpart in Eq. (31) of Ref. [16]. As pointed out at the end of Sec. II B 1, it should be stressed again that the second term in Eq. (67) represents both the ambient Casimir energy and the divergent flux on the null lines $\left(z_{ \pm}=\ell \boldsymbol{L}, \ell \in \boldsymbol{Z}\right)$ after an appropriate regularization (see Sec. IV and Appendix B), rather than a constant correction to the first divergent term.
While we have derived Eq. (67) with keeping the parallelism with the other analyses in the present paper, it is unclear from where the discrepancy comes. In the next subsection, therefore, we will rederive Eq. (67) with a method similar to the one in Ref. [16].

\section{Origin of discrepancy}

Substituting Eq. (14) into Eq. (17), and using Eq. (57), the vacuum expectation value of the energy-momentum tensor after $t=0$ is written as

$$
\begin{aligned}
\left\langle 0_{h}\left|\boldsymbol{T}_{ \pm \pm}\right| 0_{h}\right\rangle_{t>0}= & \sum_{m=1}^{\infty} \sum_{m^{\prime}=1}^{\infty}\left[\left(\left\langle 0_{h}\left|\boldsymbol{\xi}_{m} \boldsymbol{\xi}_{m^{\prime}}\right| 0_{h}\right\rangle+\sum_{k=1}^{\infty} \xi_{k m} \zeta_{k m^{\prime}}^{*}\right) \partial_{ \pm} g_{m} \partial_{ \pm} g_{m^{\prime}}\right. \\
& +\left(\left\langle 0_{h}\left|\boldsymbol{\xi}_{m} \boldsymbol{\xi}_{m^{\prime}}^{\dagger}\right| 0_{h}\right\rangle+\sum_{k=1}^{\infty} \xi_{k m} \xi_{k m^{\prime}}^{*}\right) \partial_{ \pm} g_{m} \partial_{ \pm} g_{m^{\prime}}^{*}+\left(\left\langle 0_{h}\left|\boldsymbol{\xi}_{m}^{\dagger} \boldsymbol{\xi}_{m^{\prime}}\right| 0_{h}\right\rangle+\sum_{k=1}^{\infty} \zeta_{k m} \zeta_{k m^{\prime}}^{*}\right) \partial_{ \pm} g_{m}^{*} \partial_{ \pm} g_{m^{\prime}} \\
& \left.+\left(\left\langle 0_{h}\left|\boldsymbol{\xi}_{m}^{\dagger} \boldsymbol{\xi}_{m^{\prime}}^{\dagger}\right| 0_{h}\right\rangle+\sum_{k=1}^{\infty} \zeta_{k m} \xi_{k m^{\prime}}^{*}\right) \partial_{ \pm} g_{m}^{*} \partial_{ \pm} g_{m^{\prime}}^{*}\right] .
\end{aligned}
$$


Using an explicit form of Bogoliubov coefficients (54) and (55), and mode function (8), this quantity is rewritten in a compact form,

$$
\begin{aligned}
\left\langle 0_{h}\left|\boldsymbol{T}_{ \pm \pm}\right| 0_{h}\right\rangle_{t>0}= & \frac{1}{L^{3}} \sum_{\substack{m=-\infty \\
m: \text { odd }}}^{\infty}\left(\left\langle 0_{h}\left|\boldsymbol{Q}^{2}\right| 0_{h}\right\rangle+\frac{L}{m \pi}\right) e^{-i q_{m} z_{ \pm}} \sum_{\substack{m^{\prime}=-\infty \\
m^{\prime} \text { : odd }}}^{\infty} e^{-i q_{m^{\prime}} z_{ \pm}} \\
& +\frac{1}{\pi L^{2}} \sum_{k=1}^{\infty} \frac{1}{k} \sum_{\substack{m=-\infty \\
m: \text { odd }}}^{\infty} \frac{m e^{-i\left(q_{m}-q_{k}\right) z_{ \pm}}}{m-k} \delta_{m-k: \text { odd }} \sum_{\substack{m^{\prime}=-\infty \\
m^{\prime} \text { : odd }}}^{\infty} \frac{m^{\prime} e^{-i\left(q_{m^{\prime}}+q_{k}\right) z_{ \pm}}}{m^{\prime}+k} \delta_{m^{\prime}+k \text { :odd }} .
\end{aligned}
$$

The summations over odd $m$ and $m^{\prime}$ in Eq. (69) can be evaluated with the following formulas:

$$
\begin{gathered}
\sum_{\substack{k=-\infty \\
k: \text { odd }}}^{\infty} \frac{1}{k} \exp \left(-i \frac{2 k \pi}{a} y\right)=-\frac{i \pi}{2} \sum_{\ell=-\infty}^{\infty}(-1)^{\ell} \Pi_{0}^{a / 2}\left(y-\frac{a}{2} \ell\right), \\
\sum_{\substack{k=-\infty \\
k \text { :odd }}}^{\infty} \exp \left(-i \frac{2 k \pi}{a} y\right)=\frac{a}{2} \sum_{\ell=-\infty}^{\infty}(-1)^{\ell} \delta\left(y-\frac{a}{2} \ell\right),
\end{gathered}
$$

which are equivalent to Eqs. (64) and (65), respectively.

Finally, in order to obtain the final result, it is necessary to use the following relation:

$$
\sum_{\ell=-\infty}^{\infty}(-1)^{\ell} \Pi_{0}^{L}\left(z_{ \pm}-\ell L\right) \sum_{\ell^{\prime}=-\infty}^{\infty}(-1)^{\ell^{\prime}} \Pi_{0}^{L}\left(z_{ \pm}-\ell^{\prime} L\right)= \begin{cases}0 & \left(z_{ \pm}=\ell L, \ell \in Z\right) \\ 1 & \text { (otherwise })\end{cases}
$$

Then, we obtain Eq. (67). It seems that Ref. [16] overlooked the fact that the left-hand side of Eq. (72) vanishes on null lines $z_{-}=0$ and $z_{+}=L$. This would be the origin of the discrepancy between our result and their result.

\section{SEMI-INFINITE CAVITY}

In the rest of this paper, we investigate the particle creation by the instantaneous change of boundary condition in a semi-infinite cavity, which corresponds to the limit $L \rightarrow+\infty$ of the finite-cavity model in Sec. II. We will see that some simplifications happen in such a limit. Namely, one needs just some simple integral formulas rather than the nontrivial summation formulas in Sec. II. The analysis in semi-infinite space $x \in[0,+\infty)$ can be a footing to generalize the present analysis, for example, to higher-dimensional models by regarding the spatial coordinate $x$ as a radial coordinate of higher-dimensional spaces (see [35] for a relevant higher-dimensional consideration). While the Bogoliubov transformation will be used in this section again in order to keep the parallelism with the previous sections, the results will be rederived in Appendix B with an independent method using the Green functions, which naturally involves the point-splitting regularization of the vacuum expectation value of the energymomentum tensor.

\section{A. Quantization of massless scalar field}

We consider a free massless scalar field in the semiinfinite cavity, of which equation of motion is given by Eq. (1) with $L \rightarrow+\infty$.

At left boundary $x=0$, we consider two kinds of boundary conditions. One is the Neumann boundary condition (3). Another is the Dirichlet boundary condition (4).

When the Neumann boundary condition (3) is satisfied, a natural set of positive-energy mode functions $\left\{f_{p}\right\}$, which is labeled by continuous parameter $p$, is given by

$$
f_{p}(t, x)=\frac{1}{\sqrt{\pi p}} e^{-i p t} \cos (p x), \quad p>0 .
$$

This mode function satisfies the following orthonormal conditions:

$$
\left\langle f_{p}, f_{p^{\prime}}\right\rangle=-\left\langle f_{p}^{*}, f_{p^{\prime}}^{*}\right\rangle=\delta\left(p-p^{\prime}\right), \quad\left\langle f_{p}, f_{p^{\prime}}^{*}\right\rangle=0,
$$

where the integration range of the Klein-Gordon inner product, Eq. (7), is from 0 to $+\infty$.

When the Dirichlet boundary condition (4) is satisfied, a natural set of positive-energy mode functions $\left\{g_{q}\right\}$ is given by

$$
g_{q}(t, x)=\frac{1}{\sqrt{\pi q}} e^{-i q t} \sin (q x), \quad q>0 .
$$


This mode function satisfies the following orthonormal conditions:

$$
\left\langle g_{q}, g_{q^{\prime}}\right\rangle=-\left\langle g_{q}^{*}, g_{q^{\prime}}^{*}\right\rangle=\delta\left(q-q^{\prime}\right), \quad\left\langle g_{q}, g_{q^{\prime}}^{*}\right\rangle=0 .
$$

Associated with the above two sets of mode functions, $\left\{f_{p}\right\}$ and $\left\{g_{q}\right\}$, there are two ways to quantize the scalar field. Namely, we can expand the scalar field by two sets of mode functions,

$$
\begin{aligned}
\boldsymbol{\phi} & =\int_{0}^{\infty} d p\left(\boldsymbol{a}_{p} f_{p}+\boldsymbol{a}_{p}^{\dagger} f_{p}^{*}\right), \\
\boldsymbol{\phi} & =\int_{0}^{\infty} d q\left(\boldsymbol{b}_{q} g_{q}+\boldsymbol{b}_{q}^{\dagger} g_{q}^{*}\right),
\end{aligned}
$$

where the expansion coefficients are imposed by the commutation relations,

$$
\begin{aligned}
{\left[\boldsymbol{a}_{p}, \boldsymbol{a}_{p^{\prime}}^{\dagger}\right]=\delta\left(p-p^{\prime}\right), } & {\left[\boldsymbol{a}_{p}, \boldsymbol{a}_{p^{\prime}}\right]=0, } \\
{\left[\boldsymbol{b}_{q}, \boldsymbol{b}_{q^{\prime}}^{\dagger}\right]=\delta\left(q-q^{\prime}\right), } & {\left[\boldsymbol{b}_{q}, \boldsymbol{b}_{q^{\prime}}\right]=0 . }
\end{aligned}
$$

Operators $\boldsymbol{a}_{p}$ and $\boldsymbol{b}_{q}$ (respectively, $\boldsymbol{a}_{p}^{\dagger}$ and $\boldsymbol{b}_{q}^{\dagger}$ ) are interpreted as annihilation (respectively, creation) operators.

Accordingly, we can define two normalized vacuum states,

$$
\begin{aligned}
\boldsymbol{a}_{p}\left|0_{f}\right\rangle=0, & \forall p>0, & \left\langle 0_{f} \mid 0_{f}\right\rangle=1, \\
\boldsymbol{b}_{q}\left|0_{g}\right\rangle=0, & \forall q>0, & \left\langle 0_{g} \mid 0_{g}\right\rangle=1 .
\end{aligned}
$$

Then, $\left|0_{f}\right\rangle$ (respectively, $\left|0_{g}\right\rangle$ ) is the state where no particle corresponding to $f_{n}$ (respectively, $g_{m}$ ) exists.

\section{B. Particle creation by instantaneous change of boundary condition}

Given the above quantization of the scalar field in the semi-infinite cavity, we investigate how the vacuum is excited when the boundary condition at $x=0$ instantaneously changes from Neumann to Dirichlet (N-D) in Sec. IV B 1 and reversely (D-N) in Sec. IV B 2.

\section{From Neumann to Dirichlet}

We assume that the boundary condition at $x=0$ is Neumann (3) for $t<0$ and Dirichlet (4) for $t>0$, and that the quantum field is in vacuum $\left|0_{f}\right\rangle$, defined by Eq. (81). See Fig. 5 for a schematic picture of the situation.

Let us expand $f_{p}$ by $g_{q}$ as

$$
f_{p}=\int_{0}^{\infty} d q\left(\alpha_{p q} g_{q}+\beta_{p q} g_{q}^{*}\right)
$$

where the expansion coefficients are given by

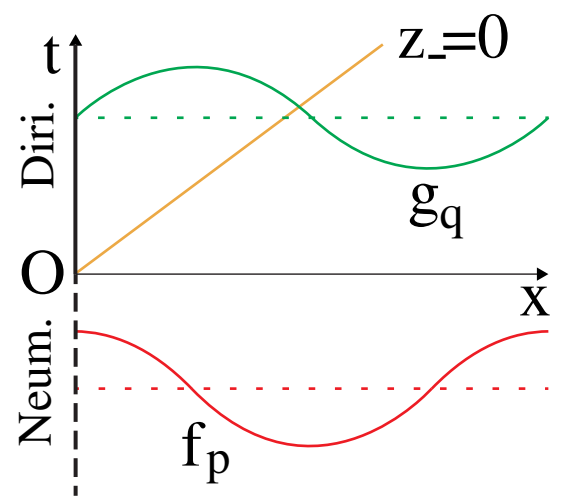

FIG. 5. The boundary condition at the left end of the domain $(x=0)$ instantaneously changes at $t=0$ from Neumann (dashed lines) to Dirichlet (solid lines). Spatial configurations of mode functions $f_{p}$ and $g_{q}$ are schematically depicted.

$$
\alpha_{p q}=\left\langle g_{q}, f_{p}\right\rangle, \quad \beta_{p q}=-\left\langle g_{q}^{*}, f_{p}\right\rangle .
$$

Using Eqs. (73) and (75), we obtain

$\alpha_{p q}=-\frac{1}{(p-q) \pi} \sqrt{\frac{q}{p}}, \quad \beta_{p q}=\frac{1}{(p+q) \pi} \sqrt{\frac{q}{p}}$,

where we have used integral formula $\int_{0}^{\infty} e^{i a x} d x=$ $i a^{-1}(-\infty<a<\infty)$.

Substituting Eq. (83) into Eq. (77), and comparing it with Eq. (78), we obtain

$$
\boldsymbol{b}_{q}=\int_{0}^{\infty} d p\left(\alpha_{p q} \boldsymbol{a}_{p}+\beta_{p q}^{*} \boldsymbol{a}_{p}^{\dagger}\right)
$$

Substituting Eq. (86) into Eq. (80), and using Eq. (79), we obtain the unitarity relations,

$$
\begin{aligned}
& \int_{0}^{\infty} d p\left(\alpha_{p q} \alpha_{p q^{\prime}}^{*}-\beta_{p q}^{*} \beta_{p q^{\prime}}\right)=\delta\left(q-q^{\prime}\right), \\
& \int_{0}^{\infty} d p\left(\alpha_{p q} \beta_{p q^{\prime}}^{*}-\beta_{p q}^{*} \alpha_{p q^{\prime}}\right)=0 .
\end{aligned}
$$

In Appendix A 4, we prove that Bogoliubov coefficients (85) satisfy Eq. (87).

The spectrum of created particles is computed as

$$
\left\langle 0_{f}\left|\boldsymbol{b}_{q}^{\dagger} \boldsymbol{b}_{q}\right| 0_{f}\right\rangle=\int_{0}^{\infty} d p\left|\beta_{p q}\right|^{2}=\frac{1}{\pi^{2}} \int_{0}^{\infty} d p \frac{q}{p(p+q)^{2}} .
$$

This and its integration over $q$ are divergent due to the contribution from the infrared regime.

The vacuum expectation value of the energy-momentum tensor before the change of boundary condition at $t=0$ is computed by substituting Eq. (77) into Eq. (17), and using Eqs. (79) and (73), as 


$$
\left\langle 0_{f}\left|\boldsymbol{T}_{ \pm \pm}\right| 0_{f}\right\rangle_{t<0}=\int_{0}^{\infty} d p\left|\partial_{ \pm} f_{p}\right|^{2}=\frac{1}{4 \pi} \int_{0}^{\infty} d p p .
$$

Unlike the finite-cavity case, there is no Casimir energy in this semi-infinite case. The above result just represents the divergent energy density due to the zero-point oscillation. Thus, the renormalized vacuum expectation value obtained by subtracting such a zero-point contribution identically vanishes everywhere as Eq. (B13).

The vacuum expectation value of the energy-momentum tensor after $t=0$ is computed by substituting Eq. (78) into Eq. (17), and using Eq. (86), as

$$
\begin{aligned}
\left\langle 0_{f}\left|\boldsymbol{T}_{ \pm \pm}\right| 0_{f}\right\rangle_{t>0}= & \int_{0}^{\infty} \int_{0}^{\infty} \int_{0}^{\infty} d p d q d q^{\prime}\left[\left(\alpha_{p q} \beta_{p q^{\prime}}\right.\right. \\
& \left.+\alpha_{p q^{\prime}} \beta_{p q}\right) \operatorname{Re}\left(\partial_{ \pm} g_{q} \partial_{ \pm} g_{q^{\prime}}\right) \\
& \left.+\left(\alpha_{p q} \alpha_{p q^{\prime}}+\beta_{p q} \beta_{p q^{\prime}}\right) \operatorname{Re}\left(\partial_{ \pm} g_{q} \partial_{ \pm} g_{q^{\prime}}^{*}\right)\right] .
\end{aligned}
$$

To derive Eq. (90), we symmetrize it with respect to integration variables $q$ and $q^{\prime}$, and use the fact that $\alpha_{p q}$ and $\beta_{p q}$ are real. Using explicit expressions of Bogoliubov coefficients (85) and mode function (75), we obtain

$$
\begin{aligned}
\left\langle 0_{f}\left|\boldsymbol{T}_{ \pm \pm}\right| 0_{f}\right\rangle_{t>0}= & \frac{1}{\pi^{3}} \int_{0}^{\infty} d p\left(\frac { 1 } { p } \left[\int_{0}^{\infty} d q \cos \left(q z_{ \pm}\right)\right.\right. \\
& \left.+p^{2} \int_{0}^{\infty} d q \frac{\cos \left(q z_{ \pm}\right)}{q^{2}-p^{2}}\right]^{2} \\
& \left.+p\left[\int_{0}^{\infty} d q \frac{q \sin \left(q z_{ \pm}\right)}{q^{2}-p^{2}}\right]^{2}\right)
\end{aligned}
$$

The integration over $q$ in Eq. (91) can be computed to give

$$
\left\langle 0_{f}\left|\boldsymbol{T}_{ \pm \pm}\right| 0_{f}\right\rangle_{t>0}=\frac{\delta^{2}\left(z_{ \pm}\right)}{\pi} \int_{0}^{\infty} \frac{d p}{p}+\frac{\operatorname{sgn}^{2}\left(z_{ \pm}\right)}{4 \pi} \int_{0}^{\infty} d p p,
$$

where sgn denotes the sign function,

$$
\operatorname{sgn}(a):=\left\{\begin{array}{ll} 
\pm 1 & (a \gtrless 0) \\
0 & (a=0)
\end{array} .\right.
$$

Note that we have used the following integration formulas:

$$
\begin{gathered}
\int_{0}^{\infty} \cos (a x) d x=\pi \delta(a) \quad(-\infty<a<\infty), \\
\int_{0}^{\infty} \frac{\cos (a x)}{x^{2}-b^{2}} d x=-\operatorname{sgn}(a) \frac{\pi}{2 b} \sin (a b) \quad(-\infty<a<\infty, b>0),
\end{gathered}
$$

$$
\int_{0}^{\infty} \frac{x \sin (a x)}{x^{2}-b^{2}} d x=\operatorname{sgn}(a) \frac{\pi}{2} \cos (a b) \quad(-\infty<a<\infty, b>0) .
$$

See Appendix C for the derivation of the second and third formulas.

Let us consider the meaning of two terms in Eq. (92). The first term, the delta function squared multiplied by a divergent integral, represents the diverging flux emanating from the origin $(t, x)=(0,0)$ and localizing on the null line $z_{-}=0$. The divergent factor involves the infrared divergence too since there is no infrared cutoff introduced by finite $L$. The dependence of energy density on the delta function squared implies also the divergence of total energy emitted.

The second term, at first glance, seems to represent an ambient divergent energy density and its vanishing on the null line emanating from the origin [note that $\operatorname{sgn}(0)=0$ ]. As will be seen below, however, this is not the case. Namely, the divergence at $z_{ \pm} \neq 0$ represents the energy due to the zero-point oscillation just as Eq. (89). Therefore, the regularized vacuum expectation value of the energymomentum tensor should be defined by subtracting such a diverging quantity distributing uniformly in space and time. As the result of such a subtraction, the divergence appears on the null line $z_{-}=0$. Such a renormalized vacuum expectation value of the energy-momentum tensor is computed in Appendix B with the Green-function method, which naturally involves the point-splitting regularization. The result is

$$
\begin{aligned}
\left\langle 0_{g}\left|\boldsymbol{T}_{ \pm \pm}\right| 0_{g}\right\rangle_{t>0}^{\mathrm{ren}}= & \frac{\delta^{2}\left(z_{ \pm}\right)}{\pi} \int_{0}^{\infty} \frac{d p}{p} \\
& + \begin{cases}\lim _{z_{ \pm}^{\prime} \rightarrow z_{ \pm}} \frac{1}{4 \pi\left(z_{ \pm}-z_{ \pm}^{\prime}\right)^{2}} & \left(z_{ \pm}=0\right) \\
0 & \text { (otherwise) }\end{cases}
\end{aligned}
$$

Here, $z_{ \pm}$and $z_{ \pm}^{\prime}$ are the coordinates of two points on which the Green functions are evaluated. As explained above, the second term diverges on the null line and vanishes elsewhere. Thus, there remain the two components of diverging flux even after the renormalization to propagate along the null line $z_{-}=0$.

\section{From Dirichlet to Neumann}

We assume that the boundary condition at $x=0$ is Dirichlet (4) for $t<0$ and Neumann (3) for $t>0$, and that the quantum field is in vacuum $\left|0_{g}\right\rangle$, given by Eq. (82). See Fig. 6 for a schematic picture of the physical situation. Then, we investigate how the vacuum is excited by computing the spectrum and energy flux of created particles. 


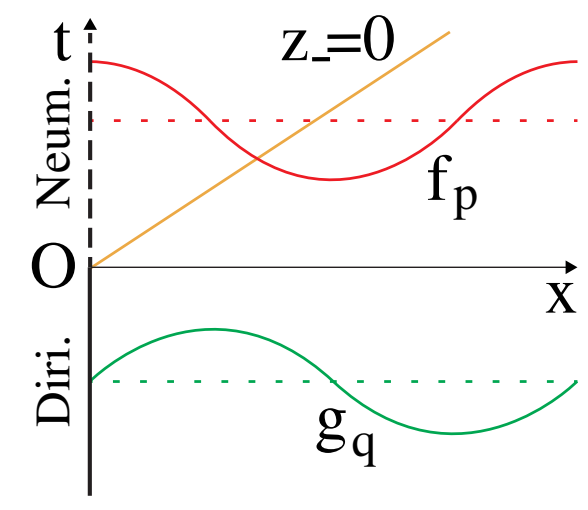

FIG. 6. The boundary condition at the left end of the domain $(x=0)$ instantaneously changes at $t=0$ from Dirichlet (solid lines) to Neumann (dashed lines). Spatial configurations of mode functions $f_{p}$ and $g_{q}$ are schematically depicted.

Let us expand $g_{q}$ by $f_{p}$ as

$$
g_{q}=\int_{0}^{\infty} d p\left(\rho_{q p} f_{p}+\sigma_{q p} f_{p}^{*}\right) .
$$

Here, the expansion coefficients are given by

$$
\rho_{q p}=\left\langle f_{p}, g_{q}\right\rangle=\alpha_{p q}^{*}, \quad \sigma_{q p}=-\left\langle f_{p}^{*}, g_{q}\right\rangle=-\beta_{p q},
$$

where $\alpha_{p q}$ and $\beta_{p q}$ are given by Eq. (85).

Substituting Eq. (98) into Eq. (78), and comparing it with Eq. (77), we obtain

$$
\boldsymbol{a}_{p}=\int_{0}^{\infty} d q\left(\rho_{q p} \boldsymbol{b}_{q}+\sigma_{q p}^{*} \boldsymbol{b}_{q}^{\dagger}\right)
$$

Substituting Eq. (100) into Eq. (79), and using Eq. (80), we obtain the unitarity relations,

$$
\begin{aligned}
& \int_{0}^{\infty} d q\left(\rho_{q p} \rho_{q p^{\prime}}^{*}-\sigma_{q p}^{*} \sigma_{q p^{\prime}}\right)=\delta\left(p-p^{\prime}\right), \\
& \int_{0}^{\infty} d q\left(\rho_{q p} \sigma_{q p^{\prime}}^{*}-\sigma_{q p}^{*} \rho_{q p^{\prime}}\right)=0 .
\end{aligned}
$$

In Appendix A 5, it is shown that Bogoliubov coefficients (99) indeed satisfy Eq. (101).

The spectrum is computed as

$$
\left\langle 0_{g}\left|\boldsymbol{a}_{p}^{\dagger} \boldsymbol{a}_{p}\right| 0_{g}\right\rangle=\int_{0}^{\infty} d q\left|\sigma_{q p}\right|^{2}=\frac{1}{\pi^{2}} \int_{0}^{\infty} d q \frac{q}{p(p+q)^{2}},
$$

which is divergent.

The expectation value of the energy-momentum tensor before the change of boundary condition at $t=0$ is computed by substituting Eq. (78) into Eq. (17), and using Eqs. (80) and (75), as

$$
\left\langle 0_{g}\left|\boldsymbol{T}_{ \pm \pm}\right| 0_{g}\right\rangle_{t<0}=\int_{0}^{\infty} d q\left|\partial_{ \pm} g_{q}\right|^{2}=\frac{1}{4 \pi} \int_{0}^{\infty} d q q .
$$

This represents the divergence due to the zero-point oscillation, and the regularized value vanishes as given by Eq. (B27).

The expectation value of the energy-momentum tensor for $t>0$ is computed by substituting Eq. (77) into Eq. (17), and using Eq. (100), as

$$
\begin{aligned}
\left\langle 0_{g}\left|\boldsymbol{T}_{ \pm \pm}\right| 0_{g}\right\rangle_{t>0}= & \int_{0}^{\infty} \int_{0}^{\infty} \int_{0}^{\infty} d q d p d p^{\prime}\left[\left(\rho_{q p} \sigma_{q p^{\prime}}\right.\right. \\
& \left.+\rho_{q p^{\prime}} \sigma_{q p}\right) \operatorname{Re}\left(\partial_{ \pm} f_{p} \partial_{ \pm} f_{p^{\prime}}\right) \\
& \left.+\left(\rho_{q p} \rho_{q p^{\prime}}+\sigma_{q p} \sigma_{q p^{\prime}}\right) \operatorname{Re}\left(\partial_{ \pm} f_{p} \partial_{ \pm} f_{p^{\prime}}^{*}\right)\right]
\end{aligned}
$$

where we symmetrize it with respect to integration variables $p$ and $p^{\prime}$, and use the fact that $\rho_{q p}$ and $\sigma_{q p}$ are real. Substituting the explicit form of the Bogoliubov coefficients, given by Eqs. (99) and (85), and mode function (75) into Eq. (104), we have

$$
\begin{aligned}
\left\langle 0_{g}\left|\boldsymbol{T}_{ \pm \pm}\right| 0_{g}\right\rangle_{t>0}= & \frac{1}{\pi^{3}} \int_{0}^{\infty} d q\left(q^{3}\left[\int_{0}^{\infty} d p \frac{\cos \left(p z_{ \pm}\right)}{p^{2}-q^{2}}\right]^{2}\right. \\
& \left.+q\left[\int_{0}^{\infty} d p \frac{p \sin \left(p z_{ \pm}\right)}{p^{2}-q^{2}}\right]^{2}\right)
\end{aligned}
$$

The integrations over $p$ in Eq. (105) are evaluated using formulas (95) and (96) to obtain

$$
\left\langle 0_{g}\left|\boldsymbol{T}_{ \pm \pm}\right| 0_{g}\right\rangle_{t>0}=\frac{\operatorname{sgn}^{2}\left(z_{ \pm}\right)}{4 \pi} \int_{0}^{\infty} d q q
$$

Again, result (106) seems to represent a diverging flux and its vanishing on the null line emanating from the origin. After subtracting the uniform contribution from the zeropoint oscillation, however, the divergence appears on the null line. This is explicitly shown by adopting the Greenfunction method in Appendix B. The result is given by

$\left\langle 0_{g}\left|\boldsymbol{T}_{ \pm \pm}\right| 0_{g}\right\rangle_{t>0}^{\text {ren }}=\left\{\begin{array}{ll}\lim _{z_{ \pm}^{\prime} \rightarrow z_{ \pm}} \frac{1}{4 \pi\left(z_{ \pm}-z_{ \pm}^{\prime}\right)^{2}} & \left(z_{ \pm}=0\right) \\ 0 & \text { (otherwise) }\end{array}\right.$.

Here, $z_{ \pm}$and $z_{ \pm}^{\prime}$ are the coordinates of two points on which the Green functions are evaluated. The flux diverges on the null line and vanishes elsewhere. Thus, there remains only one component of diverging flux after the renormalization to propagate along the null line $z_{-}=0$. 


\section{CONCLUSION}

We have investigated the particle creation due to the instantaneous change of BC in the 1D finite cavity (Secs. II and III) and semi-infinite cavity (Sec. IV) by computing the vacuum expectation value of the energy-momentum tensor for the free massless Klein-Gordon scalar field. The BC changes from Neumann to Dirichlet in Secs. II and IV, from Neumann-Neumann to Dirichlet-Dirichlet in Sec. III, and from Dirichlet to Neumann in Secs. II and IV.

Although any actual change of BC takes a finite interval of time, we believe that these models are capable of extracting the essence of the phenomenon when the $\mathrm{BC}$ changes rapidly enough compared to typical timescales in the system. In particular, it is plausible that such a situation is realized for the gravitational phenomena such as the appearance of strong (or wave-singular) naked singularities [16] and topology change of spacetime (or string) in quantum gravity [18]. In addition, the choice of Dirichlet and Neumann BCs introduced no adjustable parameters into the system, which made the whole analysis simple to be a good starting point for succeeding considerations. Most models of the particle creation due to time-dependent BCs (i.e., the dynamic Casimir effect) would have to reproduce the results in this paper in their limit of infinitely rapid change.

Thanks to the above simplifications made in our model, we could obtain almost all the results in completely analytic form. For the finite cavity N-D (respectively, D-N) case, the vacuum expectation value of the energy-momentum tensor was obtained as Eq. (34) [respectively, (48)]. Our result that the flux in the N-D and D-N cases consists of two terms and only one term, respectively, seemed to contradict the result in Ref. [16], which analyzes the NN-DD case. Therefore, we revisited the NN-DD case in Sec. III to obtain Eq. (67), which is consistent with the result in Sec. II. The flux in the N-D and NN-DD cases consist of terms of $\delta^{2}\left(z_{ \pm}\right)$and $1 /\left(z_{ \pm}-z_{ \pm}^{\prime}\right)^{2}$, while the flux in the D-N case consists of only the term of $1 /\left(z_{ \pm}-z_{ \pm}^{\prime}\right)^{2}$. Although we cannot argue which term is stronger to dominate at this point, it will be the case that not only the flux but also the total energy radiated becomes large since the integration of flux cross $z_{ \pm}=0$ diverges.

While the results in the semi-infinite cavity for the N-D case (92) and D-N case (106) are quite similar to their respective counterparts in the finite cavity, the analysis for the infinite cavity is much simpler than the finite-cavity case in that nontrivial mathematical formulas such as summation formulas of Eqs. (29), (44), and so on, are not necessary. This is a technical but an important point for succeeding studies such as the generalizations of this work (future works will be mentioned later). In addition, the vacuum expectation value of the energy-momentum tensor in the semi-infinite cavity was rederived by the Greenfunction method in Appendix B. This method not only naturally involves the point-splitting regularization but also involves only simpler calculations than the Bogoliubov method in the text. Again, this is a technical but an important point. Finally, the analysis for the semi-infinite cavity confirmed that the divergence of flux due to the change of $\mathrm{BC}$ is nothing but an ultraviolet effect rather than an infrared one, and that the divergence of the flux has nothing to do with the Casimir effect, which exists only when $L$ is finite.

Let us discuss the origin of asymmetry between the N-D and $\mathrm{D}-\mathrm{N}$ cases, of which similar conjecture was proposed in a previous paper of the present author and his collaborators [33]. The $\delta^{2}$ term seems to stem from a temporal discontinuity of mode functions $f_{n}$ and $f_{p}$. For instance, in the finite-cavity N-D case, mode function $f_{n}$ is given by Eq. (5) for $t<0$, having a nonzero value at $x=0$, but given by Eq. (18) for $t>0$, vanishing at $x=0$. Therefore, $f_{n}(t, 0)$ is discontinuous as a function of time at $t=0$. On the other hand, in the finite-cavity D-N case, mode function $g_{m}$ is given by Eq. (8) for $t<0$ and Eq. (35) for $t>0$, both of which vanish at $x=0$. Therefore, $g_{m}(t, 0)$ is continuous as a function of time at $t=0$. In a similar way, $h_{k}(t, 0)$ and $h_{k}(t, L)$ are discontinuous as functions of time at $t=0$ in the NN-DD case, and $f_{p}(t, 0)$ [respectively, $g_{q}(t, 0)$ is discontinuous (respectively, continuous) at $t=0$ in the semi-infinite N-D (respectively, D-N) case. We conjecture that such a discontinuity, which would create a shock in the classical mechanics point of view, is the origin of the delta function squared.

Naively speaking, the results in this paper suggest that the backreaction of created particles to the spacetime and/or the cavity cannot be ignored. However, the analysis is based on the test-field approximation; therefore, it is too early to assert such an implication of the results. As a next step, it is natural to investigate the backreaction through, say, the semiclassical Einstein equation, where the righthand side of the Einstein equation is replaced by the regularized vacuum expectation value of the energymomentum tensor of quantized fields [21].

Given the results in this paper, there would be several directions to proceed besides investigating the backreaction mentioned above. First, it is natural to generalize the present analysis to higher-dimensional spacetime (see Ref. [35] for a highly relevant study). Second, it would be important to generalize the $\mathrm{BC}$ in the present paper (i.e., Dirichlet and Neumann) to the Robin-type BC, which takes the form of $\phi(t, x)-\left.a \partial_{x} \phi(t, x)\right|_{x=0}=0$. Taking different values of constant $a$ before and after $t=0$, one can generalize the present analysis. By such a generalization, we would be able to verify the above conjecture about the origin of asymmetry between the N-D and D-N cases, and understand more deeply how the time-dependent BCs make the quantum vacuum excite in general.

\section{ACKNOWLEDGMENTS}

The author thanks T. Harada and S. Kinoshita for useful discussions and anonymous referees for various 
suggestions to improve the early versions of manuscript. This work was partially supported by JSPS KAKENHI Grants No. 15K05086 and No. 18K03652.

\section{APPENDIX A: Proof of unitarity relations}

\section{Equation (22)}

Using Eq. (20), the left-hand sides of Eq. (22) are written as

$$
\begin{aligned}
& \sum_{\substack{n=1 \\
n \text { :odd }}}^{\infty}\left(\alpha_{n m} \alpha_{n m^{\prime}}^{*}-\beta_{n m}^{*} \beta_{n m^{\prime}}\right)=\frac{32\left(m+m^{\prime}\right) \sqrt{m m^{\prime}}}{\pi^{2}} U_{m m^{\prime}}, \\
& \sum_{\substack{n=1 \\
n: \text { odd }}}^{\infty}\left(\alpha_{n m} \beta_{n m^{\prime}}^{*}-\beta_{n m}^{*} \alpha_{n m^{\prime}}\right)=-\frac{32\left(m-m^{\prime}\right) \sqrt{m m^{\prime}}}{\pi^{2}} U_{m m^{\prime}},
\end{aligned}
$$

where we define

$$
U_{m m^{\prime}}:=\sum_{\substack{n=1 \\ n: \text { odd }}}^{\infty} \frac{1}{\left[n^{2}-(2 m)^{2}\right]\left[n^{2}-\left(2 m^{\prime}\right)^{2}\right]}
$$

The summation over odd $n$ in Eq. (A3) can be computed to give

$$
U_{m m^{\prime}}=\frac{\pi^{2}}{16(2 m)^{2}} \delta_{m m^{\prime}},
$$

using the following formulas [34] [pp. 688 and 689],

$$
\begin{aligned}
& \sum_{k=0}^{\infty} \frac{1}{(2 k+1)^{2}-a^{2}}=\frac{\pi}{4 a} \tan \left(\frac{a \pi}{2}\right), \\
& \sum_{k=0}^{\infty} \frac{1}{\left[(2 k+1)^{2}-a^{2}\right]^{2}}=-\frac{\pi}{8 a^{3}} \tan \left(\frac{a \pi}{2}\right)+\frac{\pi^{2}}{16 a^{2}} \sec ^{2}\left(\frac{a \pi}{2}\right) .
\end{aligned}
$$

Substituting Eq. (A4) into Eqs. (A1) and (A2), we see Eq. (22) to hold.

\section{Equation (38)}

Using Eqs. (36) and (20), the left-hand sides of Eq. (38) are

$$
\begin{aligned}
& \sum_{m=1}^{\infty}\left(\rho_{m n} \rho_{m n^{\prime}}^{*}-\sigma_{m n}^{*} \sigma_{m n^{\prime}}\right)=\frac{2\left(n+n^{\prime}\right)}{\sqrt{n n^{\prime}} \pi^{2}} V_{n n^{\prime}}, \\
& \sum_{m=1}^{\infty}\left(\rho_{m n} \sigma_{m n^{\prime}}^{*}-\sigma_{m n}^{*} \rho_{m n^{\prime}}\right)=-\frac{2\left(n-n^{\prime}\right)}{\sqrt{n n^{\prime}} \pi^{2}} V_{n n^{\prime}},
\end{aligned}
$$

where we define

$$
\begin{aligned}
V_{n n^{\prime}}:= & \sum_{m=1}^{\infty} \frac{m^{2}}{\left[m^{2}-(n / 2)^{2}\right]\left[m^{2}-\left(n^{\prime} / 2\right)^{2}\right]} \\
= & \sum_{m=1}^{\infty} \frac{1}{m^{2}-\left(n^{\prime} / 2\right)^{2}}+\left(\frac{n}{2}\right)^{2} \sum_{m=1}^{\infty} \\
& \times \frac{1}{\left[m^{2}-(n / 2)^{2}\right]\left[m^{2}-\left(n^{\prime} / 2\right)^{2}\right]} .
\end{aligned}
$$

The summations over $m$ in Eq. (A9) can be computed to give

$$
V_{n n^{\prime}}=\frac{\pi^{2}}{4} \delta_{n n^{\prime}},
$$

using the following formulas [36] [pp. 68-69]:

$$
\begin{gathered}
\sum_{k=1}^{\infty} \frac{1}{y^{2}-k^{2}}=\frac{\pi}{2 y} \cot (\pi y)-\frac{1}{2 y^{2}}, \\
\sum_{k=1}^{\infty} \frac{1}{\left[(k y)^{2}-1\right]^{2}}=\frac{\pi^{2}}{4 y^{2}} \operatorname{cosec}^{2}\left(\frac{\pi}{y}\right)+\frac{\pi}{4 y} \cot \left(\frac{\pi}{y}\right)-\frac{1}{2} .
\end{gathered}
$$

Substituting Eq. (A10) into Eqs. (A7) and (A8), we see Eq. (38) to hold.

\section{Equation (58)}

Using Eqs. (51), (54), and (55), the left-hand side of Eq. (58) is written as

$$
\begin{aligned}
{\left[\boldsymbol{\xi}_{m}, \boldsymbol{\xi}_{m^{\prime}}^{\dagger}\right]+\sum_{k=1}^{\infty}\left(\xi_{k m} \xi_{k m^{\prime}}^{*}-\zeta_{k m}^{*} \zeta_{k m^{\prime}}\right) } \\
=\frac{8\left(m+m^{\prime}\right) \sqrt{m m^{\prime}}}{\pi^{2}}\left[\left(\frac{1}{2 m^{2} m^{\prime 2}}+W_{m m^{\prime}}\right) \delta_{m: \text { odd }} \delta_{m^{\prime}: \text { odd }}\right. \\
\left.\quad+X_{m m^{\prime}} \delta_{m: \text { even }} \delta_{m^{\prime}: \text { even }}\right]
\end{aligned}
$$

$$
\begin{aligned}
& {\left[\boldsymbol{\xi}_{m}, \boldsymbol{\xi}_{m^{\prime}}\right]+\sum_{k=1}^{\infty}\left(\xi_{k m} \zeta_{k m^{\prime}}^{*}-\zeta_{k m}^{*} \xi_{k m^{\prime}}\right)} \\
& =-\frac{8\left(m-m^{\prime}\right) \sqrt{m m^{\prime}}}{\pi^{2}}\left[\left(\frac{1}{2 m^{2} m^{\prime 2}}+W_{m m^{\prime}}\right) \delta_{m: \text { odd }} \delta_{m^{\prime} \text { :odd }}\right. \\
& \left.\quad+X_{m m^{\prime}} \delta_{m: \text { even }} \delta_{m^{\prime}} \text { :even }\right]
\end{aligned}
$$

where 


$$
\begin{aligned}
W_{m m^{\prime}} & :=\sum_{\substack{k=2 \\
k: \text { even }}}^{\infty} \frac{1}{\left(k^{2}-m^{2}\right)\left(k^{2}-m^{\prime 2}\right)}, \\
X_{m m^{\prime}} & :=\sum_{\substack{k=1 \\
k: \text { odd }}}^{\infty} \frac{1}{\left(k^{2}-m^{2}\right)\left(k^{2}-m^{\prime 2}\right)} .
\end{aligned}
$$

Applying formulas (A11) and (A12) to $W_{m m^{\prime}}$ and formulas (A5) and (A6) to $X_{m m^{\prime}}$, we obtain

$$
W_{m m^{\prime}}=-\frac{1}{2 m^{2} m^{\prime 2}}+\frac{\pi^{2}}{16 m^{2}} \delta_{m m^{\prime}}, \quad X_{m m^{\prime}}=\frac{\pi^{2}}{16 m^{2}} \delta_{m m^{\prime}}
$$

Substituting Eq. (A16) into Eqs. (A13) and (A14), we see that unitarity relation (58) holds.

\section{Equation (87)}

Using Eq. (85), the left-hand side of Eq. (87) is written as

$$
\int_{0}^{\infty} d p\left(\alpha_{p q} \alpha_{p q^{\prime}}^{*}-\beta_{p q}^{*} \beta_{p q^{\prime}}\right)=\frac{2\left(q+q^{\prime}\right) \sqrt{q q^{\prime}}}{\pi^{2}} U_{q q^{\prime}},
$$

$$
\int_{0}^{\infty} d p\left(\alpha_{p q} \beta_{p q^{\prime}}^{*}-\beta_{p q}^{*} \alpha_{p q^{\prime}}\right)=-\frac{2\left(q-q^{\prime}\right) \sqrt{q q^{\prime}}}{\pi^{2}} U_{q q^{\prime}},
$$

where we define

$$
U_{q q^{\prime}}:=\int_{0}^{\infty} \frac{d p}{\left(p^{2}-q^{2}\right)\left(p^{2}-q^{\prime 2}\right)}
$$

By simple algebra, this is rewritten as

$$
\begin{aligned}
U_{q q^{\prime}}= & \frac{1}{4 q\left(q+q^{\prime}\right)} \int_{-\infty}^{\infty} d p\left[\frac{1}{(p-q)\left(p-q^{\prime}\right)}\right. \\
& \left.+\frac{1}{(p+q)\left(p+q^{\prime}\right)}-\frac{2}{p^{2}-q^{\prime 2}}\right] .
\end{aligned}
$$

Adapting the following formula [37] [p. 488] to the first and second terms of Eq. (A20),

$$
\int_{-\infty}^{\infty} \frac{d x}{(x-a)(x-b)}=\pi^{2} \delta(a-b) \quad(-\infty<a, b<\infty),
$$

and noting the third term vanishes from Eq. (95), we have

$$
U_{q q^{\prime}}=\frac{\pi^{2}}{4 q^{2}} \delta\left(q-q^{\prime}\right)
$$

Substituting Eq. (A22) into Eqs. (A17) and (A18), we see Eq. (87) to hold.

\section{Equation (101)}

Using Eqs. (99) and (85), the left-hand side of Eq. (101) is written as

$$
\begin{aligned}
& \int_{0}^{\infty} d q\left(\rho_{q p} \rho_{q p^{\prime}}^{*}-\sigma_{q p}^{*} \sigma_{q p^{\prime}}\right)=\frac{2\left(p+p^{\prime}\right)}{\pi^{2} \sqrt{p p^{\prime}}} V_{p p^{\prime}}, \\
& \int_{0}^{\infty} d q\left(\rho_{q p} \sigma_{q p^{\prime}}^{*}-\sigma_{q p}^{*} \rho_{q p^{\prime}}\right)=-\frac{2\left(p-p^{\prime}\right)}{\pi^{2} \sqrt{p p^{\prime}}} V_{p p^{\prime}},
\end{aligned}
$$

where we define

$$
V_{p p^{\prime}}:=\int_{0}^{\infty} d q \frac{q^{2}}{\left(q^{2}-p^{2}\right)\left(q^{2}-p^{\prime 2}\right)}
$$

This is computed as

$$
\begin{aligned}
V_{p p^{\prime}} & =\int_{0}^{\infty} d q \frac{1}{q^{2}-p^{\prime 2}}+p^{2} \int_{0}^{\infty} d q \frac{1}{\left(q^{2}-p^{2}\right)\left(q^{2}-p^{\prime 2}\right)} \\
& =\frac{\pi^{2}}{4} \delta\left(p-p^{\prime}\right),
\end{aligned}
$$

where the first term vanishes from Eq. (95), and the technique to obtain Eq. (A22) is used to compute the second term. Substituting Eq. (A26) into Eqs. (A23) and (A24), we see Eq. (101) to hold.

\section{APPENDIX B: Green-function method for semi-infinite cavity}

We reanalyze the vacuum excitation by the change of boundary condition for the semi-infinite cavity using the Green-function method [21,33], which naturally incorporates the renormalization of zero-point energy.

\section{Green functions}

Two Hadamard elementary functions, $F^{(1)}$ and $G^{(1)}$, are defined by

$$
\begin{aligned}
& F^{(1)}\left(z, z^{\prime}\right):=\left\langle 0_{f}\left|\left\{\boldsymbol{\phi}(z), \boldsymbol{\phi}\left(z^{\prime}\right)\right\}\right| 0_{f}\right\rangle, \\
& G^{(1)}\left(z, z^{\prime}\right):=\left\langle 0_{g}\left|\left\{\boldsymbol{\phi}(z), \boldsymbol{\phi}\left(z^{\prime}\right)\right\}\right| 0_{g}\right\rangle,
\end{aligned}
$$

where we have introduced a simplified notation $z:=$ $\left(z_{-}, z_{+}\right)$and $z^{\prime}:=\left(z_{-}^{\prime}, z_{+}^{\prime}\right)$, and $\{\cdot, \cdot\}$ denotes the anticommutator, $\{\phi, \psi\}:=\phi \psi+\psi \phi$. Two Pauli-Jordan or Schwinger functions, $F$ and $G$, are defined by 


$$
\begin{aligned}
i F\left(z, z^{\prime}\right) & :=\left\langle 0_{f}\left|\left[\boldsymbol{\phi}(z), \boldsymbol{\phi}\left(z^{\prime}\right)\right]\right| 0_{f}\right\rangle, \\
i G\left(z, z^{\prime}\right) & :=\left\langle 0_{g}\left|\left[\boldsymbol{\phi}(z), \boldsymbol{\phi}\left(z^{\prime}\right)\right]\right| 0_{g}\right\rangle .
\end{aligned}
$$

Using the decompositions of field operators (77) and (78), the Hadamard elementary functions are represented as

$$
\begin{aligned}
F^{(1)}\left(z, z^{\prime}\right)= & \int_{0}^{\infty} d p\left[f_{p}(z) f_{p}^{*}\left(z^{\prime}\right)+\text { c.c. }\right] \\
= & \frac{1}{2 \pi} \int_{0}^{\infty} \frac{d p}{p}\left[\cos \left(p \Delta z_{-}\right)+\cos \left(p \Delta z_{+}\right)\right. \\
& \left.+\cos p\left(z_{-}-z_{+}{ }^{\prime}\right)+\cos p\left(z_{+}-z_{-}{ }^{\prime}\right)\right], \\
G^{(1)}\left(z, z^{\prime}\right)= & \int_{0}^{\infty} d q\left[g_{q}(z) g_{q}^{*}\left(z^{\prime}\right)+\text { c.c. }\right] \\
= & \frac{1}{2 \pi} \int_{0}^{\infty} \frac{d q}{q}\left[\cos \left(q \Delta z_{-}\right)+\cos \left(q \Delta z_{+}\right)\right. \\
& \left.-\cos q\left(z_{-}-z_{+}{ }^{\prime}\right)-\cos q\left(z_{+}-z_{-}{ }^{\prime}\right)\right],
\end{aligned}
$$

where c.c. denotes the complex conjugate and $\Delta z_{ \pm}:=$ $z_{ \pm}-z_{ \pm}^{\prime}$. For the Pauli-Jordan functions, the momentum integration can be evaluated to give

$$
\begin{aligned}
i F\left(z, z^{\prime}\right)= & -\frac{i}{4}\left[\operatorname{sgn}\left(\Delta z_{-}\right)+\operatorname{sgn}\left(\Delta z_{+}\right)\right. \\
& \left.+\operatorname{sgn}\left(z_{-}-z_{+}^{\prime}\right)+\operatorname{sgn}\left(z_{+}-z_{-}^{\prime}\right)\right], \\
i G\left(z, z^{\prime}\right)= & -\frac{i}{4}\left[\operatorname{sgn}\left(\Delta z_{-}\right)+\operatorname{sgn}\left(\Delta z_{+}\right)\right. \\
& \left.-\operatorname{sgn}\left(z_{-}-z_{+}^{\prime}\right)-\operatorname{sgn}\left(z_{+}-z_{-}^{\prime}\right)\right],
\end{aligned}
$$

where we have used $\int_{0}^{\infty} \frac{\sin (a x)}{x} d x= \pm \frac{\pi}{2}(a \gtrless 0)$ [p. 251].

\section{From Neumann to Dirichlet}

The vacuum expectation value of the energy-momentum tensor before the change of boundary condition is obtained by differentiating the Hadamard elementary function $F^{(1)}$ with respect to two points $z$ and $z^{\prime}$, and taking the samepoint limit $z^{\prime} \rightarrow z$,

$$
\left\langle 0_{f}\left|\boldsymbol{T}_{ \pm \pm}\right| 0_{f}\right\rangle_{t<0}^{\text {Green }}=\frac{1}{2} \lim _{z^{\prime} \rightarrow z} \partial_{ \pm} \partial_{ \pm}^{\prime} F^{(1)}\left(z, z^{\prime}\right) .
$$

From Eqs. (B4) and (B9), one obtains

$$
\begin{aligned}
\left\langle 0_{f}\left|\boldsymbol{T}_{ \pm \pm}\right| 0_{f}\right\rangle_{t<0}^{\text {Green }}= & \lim _{z^{\prime} \rightarrow z} \frac{1}{4 \pi} \int_{0}^{\infty} d p p \cos \left(p \Delta z_{ \pm}\right) \\
& =\lim _{z^{\prime} \rightarrow z} \frac{-1}{4 \pi\left(\Delta z_{ \pm}\right)^{2}} .
\end{aligned}
$$

One can see that Eq. (B10) reproduces Eq. (89) if one takes limit $z^{\prime} \rightarrow z$ before the $p$ integration. Equation (B11) shows that $\left\langle 0_{f}\left|\boldsymbol{T}_{ \pm \pm}\right| 0_{f}\right\rangle_{t<0}^{\text {Green }}$ contains the ultraviolet divergence $\sim 1 /\left(\Delta z_{ \pm}\right)^{2}$, which is the vacuum energy due to the zeropoint oscillation always existing even in a free Mankowski spacetime. Therefore, the renormalized energy-momentum is defined by subtracting this ultraviolet divergence as

$$
\begin{aligned}
\left\langle 0_{f}\left|\boldsymbol{T}_{ \pm \pm}\right| 0_{f}\right\rangle_{t<0}^{\text {ren }} & :=\left\langle 0_{f}\left|\boldsymbol{T}_{ \pm \pm}\right| 0_{f}\right\rangle_{t<0}^{\text {Green }}-\lim _{z^{\prime} \rightarrow z} \frac{-1}{4 \pi\left(\Delta z_{ \pm}\right)^{2}} \\
& =0
\end{aligned}
$$

which reasonably vanishes before changing the boundary condition.

The vacuum expectation value of the energy-momentum tensor after the change of the boundary condition has the same expression as Eq. (B9). However, since the boundary condition is changed at $t=0$, Hadamard elementary function $F^{(1)}$ before the change of the boundary condition has to be propagated into the $t>0$ region using Pauli-Jordan function $i G$ [33]. Thus, the energy momentum is represented as

$$
\begin{aligned}
& \left\langle 0_{f}\left|\boldsymbol{T}_{ \pm \pm}\right| 0_{f}\right\rangle_{t>0}^{\text {Green }} \\
& \quad=\frac{1}{2} \lim _{B \rightarrow A} \partial_{ \pm} \partial_{ \pm}^{\prime}\left[i G(A, C) i G(B, D) F^{(1)}(C, D)\right],
\end{aligned}
$$

where $A:=z$ and $B:=z^{\prime}$. Namely, in this abbreviated notation, let capital Latin letters (except $G$ and $F$ ) denote a world point, e.g., $\phi(A, B)=\phi\left(z, z^{\prime}\right)$. In addition, let a pair of repeated capital Latin letters denote the KleinGordon inner product at $t=0$, e.g., $\phi(A) \psi(A):=\left.\langle\phi, \psi\rangle\right|_{t=0}$.

Substituting Eq. (B3) into Eq. (B14), one obtains

$$
\begin{aligned}
\left\langle 0_{f}\left|\boldsymbol{T}_{ \pm \pm}\right| 0_{f}\right\rangle_{t>0}^{\text {Green }}= & \frac{1}{2} \lim _{B \rightarrow A} \int_{0}^{\infty} d p\left(i \partial_{ \pm} G(A, C) f_{p}(C)\right. \\
& \left.\times\left[i \partial_{ \pm}^{\prime} G(B, D) f_{p}(D)\right]^{*}+\text { c.c. }\right),
\end{aligned}
$$

where we have used the property of inner product $\left\langle\phi, \psi^{*}\right\rangle=-\left\langle\phi^{*}, \psi\right\rangle^{*}$. The inner product in Eq. (B15) can be written as

$$
\begin{aligned}
i \partial_{ \pm} G(A, B) f_{p}(B)= & \int_{0}^{\infty} d x^{\prime}\left[\partial_{ \pm} G\left(z, z^{\prime}\right) \partial_{t^{\prime}} f_{p}\left(z^{\prime}\right)\right. \\
& \left.-\partial_{t^{\prime}} \partial_{ \pm} G\left(z, z^{\prime}\right) f_{p}\left(z^{\prime}\right)\right]\left.\right|_{t^{\prime}=0} .
\end{aligned}
$$

Using Eq. (B8), derivatives of $G$ in Eq. (B16) are computed as

$$
\begin{aligned}
\left.\partial_{ \pm} G\left(z, z^{\prime}\right)\right|_{t^{\prime}=0} & =-\frac{1}{2}\left[\delta\left(x^{\prime} \mp z_{ \pm}\right)-\delta\left(x^{\prime} \pm z_{ \pm}\right)\right], \\
\left.\partial_{t^{\prime}} \partial_{ \pm} G\left(z, z^{\prime}\right)\right|_{t^{\prime}=0} & =\mp \frac{1}{2} \partial_{x^{\prime}}\left[\delta\left(x^{\prime} \mp z_{ \pm}\right)+\delta\left(x^{\prime} \pm z_{ \pm}\right)\right],
\end{aligned}
$$


where $\operatorname{sgn}^{\prime}(x)=2 \delta(x)$ was used. Substituting Eqs. (B17) and (B18) into Eq. (B16), one obtains

$i \partial_{ \pm} G(A, B) f_{p}(B)= \pm \frac{i}{2} \sqrt{\frac{p}{\pi}} \operatorname{sgn}\left(z_{ \pm}\right) e^{-i p z_{ \pm}} \mp \frac{\delta\left(z_{ \pm}\right)}{\sqrt{\pi p}}$,

where we have used

$$
\begin{gathered}
\int_{0}^{\infty} \delta(x-a) f(x) d x=\theta(a) f(a), \\
\theta( \pm x)-\theta(\mp x)= \pm \operatorname{sgn}(x) .
\end{gathered}
$$

With Eq. (B19), Eq. (B15) yields

$$
\begin{aligned}
\left\langle 0_{f}\left|\boldsymbol{T}_{ \pm \pm}\right| 0_{f}\right\rangle_{t>0}^{\text {Green }}= & \frac{\delta^{2}\left(z_{ \pm}\right)}{\pi} \int_{0}^{\infty} \frac{d p}{p} \\
& +\operatorname{sgn}^{2}\left(z_{ \pm}\right) \lim _{z^{\prime} \rightarrow z} \frac{-1}{4 \pi\left(\Delta z_{ \pm}\right)^{2}} .
\end{aligned}
$$

The last term in Eq. (B22) shows that $\left\langle 0_{g}\left|\boldsymbol{T}_{ \pm \pm}\right| 0_{g}\right\rangle_{t>0}^{\text {Green }}$ contains the ultraviolet divergence due to zero-point oscillation. Thus, the renormalized energy momentum is defined in the same way as Eq. (B12) by subtracting the zero-point energy,

$$
\begin{aligned}
& \left\langle 0_{f}\left|\boldsymbol{T}_{ \pm \pm}\right| 0_{f}\right\rangle_{t>0}^{\text {ren }}:=\left\langle 0_{f}\left|\boldsymbol{T}_{ \pm \pm}\right| 0_{f}\right\rangle_{t>0}^{\text {Green }}-\lim _{z^{\prime} \rightarrow z} \frac{-1}{4 \pi\left(\Delta z_{ \pm}\right)^{2}} \\
& =\frac{\delta^{2}\left(z_{ \pm}\right)}{\pi} \int_{0}^{\infty} \frac{d p}{p}+ \begin{cases}\lim _{z^{\prime} \rightarrow z} \frac{1}{4 \pi\left(\Delta z_{ \pm}\right)^{2}} & \left(z_{ \pm}=0\right) \\
0 & \text { (otherwise) }\end{cases}
\end{aligned}
$$

which is nothing but Eq. (97).

\section{From Dirichlet to Neumann}

The vacuum expectation value of the energy-momentum tensor before the change of the boundary condition is given by

$$
\left\langle 0_{g}\left|\boldsymbol{T}_{ \pm \pm}\right| 0_{g}\right\rangle_{t<0}^{\text {Green }}=\frac{1}{2} \lim _{z^{\prime} \rightarrow z} \partial_{ \pm} \partial_{ \pm}^{\prime} G^{(1)}\left(z, z^{\prime}\right) .
$$

Substituting Eq. (B6) into Eq. (B25), one obtains

$$
\begin{aligned}
\left\langle 0_{g}\left|\boldsymbol{T}_{ \pm \pm}\right| 0_{g}\right\rangle_{t<0}^{\text {Green }} & =\lim _{z^{\prime} \rightarrow z} \frac{1}{4 \pi} \int_{0}^{\infty} d q q \cos \left(q \Delta z_{ \pm}\right) \\
& =\lim _{z^{\prime} \rightarrow z} \frac{-1}{4 \pi\left(\Delta z_{ \pm}\right)^{2}} .
\end{aligned}
$$

This represents the ultraviolet divergence due to the zeropoint oscillation. The normalized energy momentum is defined by subtracting such a divergence,

$$
\left\langle 0_{g}\left|\boldsymbol{T}_{ \pm \pm}\right| 0_{g}\right\rangle_{t<0}^{\text {ren }}:=\left\langle 0_{g}\left|\boldsymbol{T}_{ \pm \pm}\right| 0_{g}\right\rangle_{t<0}^{\text {Green }}-\lim _{z^{\prime} \rightarrow z} \frac{-1}{4 \pi\left(\Delta z_{ \pm}\right)^{2}}=0,
$$

which reasonably vanishes before the change of boundary condition.

The energy momentum after the change of boundary condition is obtained by propagating $G^{(1)}$ by $i F$,

$$
\begin{aligned}
& \left\langle 0_{g}\left|\boldsymbol{T}_{ \pm \pm}\right| 0_{g}\right\rangle_{t>0}^{\text {Green }} \\
& \quad=\frac{1}{2} \lim _{B \rightarrow A} \partial_{ \pm} \partial_{ \pm}{ }^{\prime}\left[i F(A, C) i F(B, D) G^{(1)}(C, D)\right] .
\end{aligned}
$$

Substituting Eq. (B5), this quantity is represented as

$$
\begin{aligned}
\left\langle 0_{g}\left|\boldsymbol{T}_{ \pm \pm}\right| 0_{g}\right\rangle_{t>0}^{\text {Green }}= & \frac{1}{2} \lim _{B \rightarrow A} \int_{0}^{\infty} d q\left(i \partial_{ \pm} F(A, C) g_{q}(C)\right. \\
& \left.\times\left[i \partial_{ \pm}^{\prime} F(B, D) g_{q}(D)\right]^{*}+\text { c.c. }\right) .
\end{aligned}
$$

The inner product in Eq. (B29) is written as

$$
\begin{aligned}
i \partial_{ \pm} & F(A, B) g_{q}(B) \\
\quad= & \left.\int_{0}^{\infty} d x^{\prime}\left[\partial_{ \pm} F\left(z, z^{\prime}\right) \partial_{t^{\prime}} g_{q}\left(z^{\prime}\right)-\partial_{t^{\prime}} \partial_{ \pm} F\left(z, z^{\prime}\right) g_{q}\left(z^{\prime}\right)\right]\right|_{t^{\prime}=0} .
\end{aligned}
$$

Using Eq. (B7), derivatives of $F$ in Eq. (B30) are computed as

$\left.\partial_{ \pm} F\left(z, z^{\prime}\right)\right|_{t^{\prime}=0}=-\frac{1}{2}\left[\delta\left(x^{\prime} \mp z_{ \pm}\right)+\delta\left(x^{\prime} \pm z_{ \pm}\right)\right]$,

$\left.\partial_{t^{\prime}} \partial_{ \pm} F\left(z, z^{\prime}\right)\right|_{t^{\prime}=0}=\mp \frac{1}{2} \partial_{x^{\prime}}\left[\delta\left(x^{\prime} \mp z_{ \pm}\right)-\delta\left(x^{\prime} \pm z_{ \pm}\right)\right]$.

Substitution of Eqs. (B31) and (B32) into Eq. (B30) yields

$$
i \partial_{ \pm} F(A, B) g_{q}(B)=-\frac{1}{2} \sqrt{\frac{q}{\pi}} \operatorname{sgn}\left(z_{ \pm}\right) e^{-i q z_{ \pm}},
$$

where we have used formulas (B20) and (B21). The combination of Eqs. (B33) and (B29) gives

$$
\left\langle 0_{g}\left|\boldsymbol{T}_{ \pm \pm}\right| 0_{g}\right\rangle_{t>0}^{\text {Green }}=\operatorname{sgn}^{2}\left(z_{ \pm}\right) \lim _{z^{\prime} \rightarrow z} \frac{-1}{4 \pi\left(\Delta z_{ \pm}\right)^{2}} .
$$

The renormalized energy momentum is obtained by subtracting the zero-point energy (B26) from Eq. (B34), 


$$
\begin{aligned}
\left\langle 0_{g}\left|\boldsymbol{T}_{ \pm \pm}\right| 0_{g}\right\rangle_{t>0}^{\text {ren }} & :=\left\langle 0_{g}\left|\boldsymbol{T}_{ \pm \pm}\right| 0_{g}\right\rangle_{t>0}^{\text {Green }}-\lim _{z^{\prime} \rightarrow z} \frac{-1}{4 \pi\left(\Delta z_{ \pm}\right)^{2}} \\
& = \begin{cases}\lim _{z^{\prime} \rightarrow z} \frac{1}{4 \pi\left(\Delta z_{ \pm}\right)^{2}} & \left(z_{ \pm}=0\right) \\
0 & \text { (otherwise) }\end{cases}
\end{aligned}
$$

which is nothing but Eq. (107).

\section{APPENDIX C: Integral formulas (95) and (96)}

Let us calculate the principal values of the following integrals:

$$
I:=\int_{0}^{\infty} \frac{\cos (a x)}{x^{2}-b^{2}} d x, \quad J:=\int_{0}^{\infty} \frac{x \sin (a x)}{x^{2}-b^{2}} d x
$$

where $-\infty<a<\infty, b>0$. Note that we always consider only principal values for improper integrals. These are written as

$$
\begin{gathered}
I=\frac{1}{4}\left(I_{+}+I_{-}\right), \quad I_{ \pm}:=\int_{-\infty}^{\infty} \frac{e^{ \pm i a x}}{x^{2}-b^{2}} d x, \\
J=\frac{1}{4 i}\left(J_{+}-J_{-}\right), \quad J_{ \pm}:=\int_{-\infty}^{\infty} \frac{x e^{ \pm i a x}}{x^{2}-b^{2}} .
\end{gathered}
$$

We suppose two contours $C_{+}$and $C_{-}$drawn in Fig. 7 and use Cauchy's integral theorem and the residue theorem.

For $a>0$, taking contour $C_{ \pm}$for $I_{ \pm}$and $J_{ \pm}$, we have

$$
\begin{aligned}
0= & \int_{C_{ \pm}} \frac{e^{ \pm i a z}}{z^{2}-b^{2}} d z=I_{ \pm} \mp \frac{1}{2} \cdot 2 \pi i \operatorname{Res}\left[I_{ \pm},-b\right] \\
& \mp \frac{1}{2} \cdot 2 \pi i \operatorname{Res}\left[I_{ \pm}, b\right], \\
0= & \int_{C_{ \pm}} \frac{z e^{ \pm i a z}}{z^{2}-b^{2}} d z=J_{ \pm} \mp \frac{1}{2} \cdot 2 \pi i \operatorname{Res}\left[J_{ \pm},-b\right] \\
& \mp \frac{1}{2} \cdot 2 \pi i \operatorname{Res}\left[J_{ \pm}, b\right] .
\end{aligned}
$$

Here, $\operatorname{Res}\left[X, z_{0}\right]$ denotes the residue of the integrand of $X$ at $z=z_{0}$, and the contributions from the large semicircles vanish from Jordan's lemma. Substituting the following values of residues,

$$
\begin{aligned}
& \operatorname{Res}\left[I_{ \pm},-b\right]=-\frac{e^{\mp i a b}}{2 b}, \quad \operatorname{Res}\left[I_{ \pm}, b\right]=\frac{e^{ \pm i a b}}{2 b}, \\
& \operatorname{Res}\left[J_{ \pm},-b\right]=\frac{e^{\mp i a b}}{2}, \quad \operatorname{Res}\left[J_{ \pm}, b\right]=\frac{e^{ \pm i a b}}{2}
\end{aligned}
$$

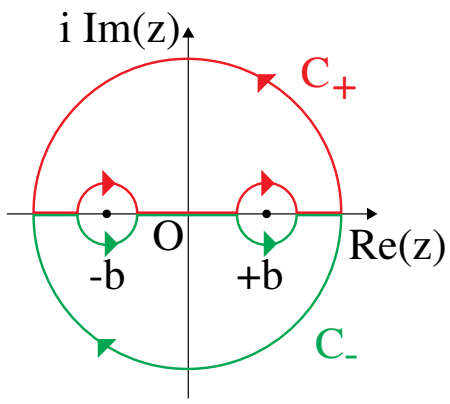

FIG. 7. Two closed contours $C_{+}$and $C_{-}$in the complex plane, each of which contains an infinitely large semicircle and two infinitesimal semicircles to avoid $-b$ and $+b$ on the real axis.

into Eqs. (C4) and (C5), we have

$I_{ \pm}=-\frac{\pi}{b} \sin (a b), \quad J_{ \pm}= \pm i \pi \cos (a b) \quad(a>0)$.

For $a<0$, taking contour $C_{\mp}$ for $I_{ \pm}$and $J_{ \pm}$, we have

$$
\begin{aligned}
0= & \int_{C_{\mp}} \frac{e^{ \pm i a z}}{z^{2}-b^{2}} d z=I_{ \pm} \pm \frac{1}{2} \cdot 2 \pi i \operatorname{Res}\left[I_{ \pm},-b\right] \\
& \pm \frac{1}{2} \cdot 2 \pi i \operatorname{Res}\left[I_{ \pm}, b\right]
\end{aligned}
$$

$$
\begin{aligned}
0= & \int_{C_{\mp}} \frac{z e^{ \pm i a z}}{z^{2}-b^{2}} d z=J_{ \pm} \pm \frac{1}{2} \cdot 2 \pi i \operatorname{Res}\left[J_{ \pm},-b\right] \\
& \pm \frac{1}{2} \cdot 2 \pi i \operatorname{Res}\left[J_{ \pm}, b\right]
\end{aligned}
$$

Using Eq. (C6) again, we have

$$
I_{ \pm}=\frac{\pi}{b} \sin (a b), \quad J_{ \pm}=\mp i \pi \cos (a b) \quad(a<0) .
$$

For $a=0$, taking either $C_{+}$or $C_{-}$for $I_{ \pm}$, one can show that $I_{ \pm}$vanishes. In addition, $J$ obviously vanishes by definition (C1). Thus, we have

$$
I_{ \pm}=J=0 \quad(a=0) .
$$

Combining Eqs. (C2), (C3), (C7), (C10), and (C11), we see formulas (95) and (96) to hold. 
[1] H. B. G. Casimir, On the attraction between two perfectly conducting plates, Proc. K. Ned. Akad. Wet. 51, 793 (1948).

[2] Casimir Physics, edited by D. A. R. Dalvit, P. Milonni, D. Roberts, and F. Rosa, Lecture Notes in Physics Vol. 834 (Springer, 2011), p. 457.

[3] G. T. Moore, Quantum theory of the electromagnetic field in a variable-length one-dimensional cavity, J. Math. Phys. (N.Y.) 11, 2679 (1970).

[4] D. A. R. Dalvit, P. A. Maia Neto, and F. D. Mazzitelli, Fluctuations, dissipation and the dynamical Casimir effect, Lect. Notes Phys. 834, 419 (2011).

[5] C. M. Wilson, G. Johansson, A. Pourkabirian, M. Simonen, J. R. Johansson, T. Duty, F. Nori, and P. Delsing, Observation of the dynamical Casimir effect in a superconducting circuit, Nature (London) 479, 376 (2011).

[6] V. V. Dodonov, Current status of the dynamical Casimir effect, Phys. Scr. 82, 038105 (2010).

[7] P. D. Nation, J. R. Johansson, M. P. Blencowe, and F. Nori, Stimulating uncertainty: Amplifying the quantum vacuum with superconducting circuits, Rev. Mod. Phys. 84, 1 (2012).

[8] C. Farina, H. O. Silva, A. L. C. Rego, and D. T. Alves, Timedependent Robin boundary conditions in the dynamical Casimir effect, Int. J. Mod. Phys. Conf. Ser. 14, 306 (2012).

[9] V. V. Dodonov, Nonstationary Casimir effect and analytical solutions for quantum fields in cavities with moving boundaries, Adv. Chem. Phys. 119, 309 (2001).

[10] A. Mitra, Quantum quench dynamics, Annu. Rev. Condens. Matter Phys. 9, 245 (2018).

[11] W. Berdanier, M. Kolodrubetz, R. Vasseur, and J. E. Moore, Floquet Dynamics of Boundary-Driven Systems at Criticality, Phys. Rev. Lett. 118, 260602 (2017).

[12] S. He, T. Numasawa, T. Takayanagi, and K. Watanabe, Quantum dimension as entanglement entropy in two dimensional conformal field theories, Phys. Rev. D 90, 041701 (2014).

[13] L. Parker, Particle creation in expanding universes, Phys. Rev. Lett. 21, 562 (1968).

[14] S. W. Hawking, Particle creation by black holes, Commun. Math. Phys. 43, 199 (1975); Erratum, Commun. Math. Phys. 46, 206(E) (1976).

[15] L. H. Ford and L. Parker, Creation of particles by singularities in asymptotically flat space-times, Phys. Rev. D 17, 1485 (1978).

[16] A. Ishibashi and A. Hosoya, Naked singularity and thunderbolt, Phys. Rev. D 66, 104016 (2002).

[17] S. L. Braunstein, A toy model for slowly growing wormholes as effective topology changes, arXiv:gr-qc/9610056.

[18] A. Anderson and B. S. DeWitt, Does the topology of space fluctuate?, Found. Phys. 16, 91 (1986).

[19] C. A. Manogue, E. Copeland, and T. Dray, The trousers problem revisited, Pramana 30, 279 (1998).

[20] A. D. Shapere, F. Wilczek, and Z. Xiong, Models of topology change, arXiv:1210.3545.
[21] N. D. Birrell and P.C. W. Davies, Quantum Fields in Curved Space (Cambridge University Press, Cambridge, England, 1984).

[22] R. M. Wald, Quantum Field Theory in Curved Space-Time and Black Hole Thermodynamics (Chicago University Press, Chicago, 1994).

[23] R. Penrose, Gravitational collapse: The role of general relativity, Riv. Nuovo Cimento 1, 252 (1969); Gen. Relativ. Gravit. 34, 1141 (2002).

[24] S. W. Hawking and G. F. R. Ellis, The Large Scale Structure of Space-Time (Cambridge University Press, Cambridge, 1973).

[25] R. M. Wald, Dynamics in nonglobally hyperbolic, static space-times, J. Math. Phys. (N.Y.) 21, 2802 (1980).

[26] G. T. Horowitz and D. Marolf, Quantum probes of spacetime singularities, Phys. Rev. D 52, 5670 (1995).

[27] A. Ishibashi and A. Hosoya, Who's afraid of naked singularities? Probing timelike singularities with finite energy waves, Phys. Rev. D 60, 104028 (1999).

[28] A. Romeo and A. A. Saharian, Casimir effect for scalar fields under Robin boundary conditions on plates, J. Phys. A 35, 1297 (2002).

[29] B. Mintz, C. Farina, P. A. Maia Neto, and R. B. Rodrigues, Casimir forces for moving boundaries with Robin conditions, J. Phys. A 39, 6559 (2006).

[30] B. Mintz, C. Farina, P. A. Maia Neto, and R. B. Rodrigues, Particle creation by a moving boundary with Robin boundary condition, J. Phys. A 39, 11325 (2006).

[31] M. R. Vazquez, M. del Rey, H. Westman, and J. Leon, Local quanta, unitary inequivalence, and vacuum entanglement, Ann. Phys. (Amsterdam) 351, 112 (2014).

[32] E. G. Brown and J. Louko, Smooth and sharp creation of a Dirichlet wall in $1+1$ quantum field theory: How singular is the sharp creation limit?, J. High Energy Phys. 08 (2015) 061.

[33] T. Harada, S. Kinoshita, and U. Miyamoto, Vacuum excitation by sudden appearance and disappearance of a Dirichlet wall in a cavity, Phys. Rev. D 94, 025006 (2016).

[34] Y. Otsuki and Y. Muroya, Shin Sugaku Koshiki Shu, in Japanese (Maruzen Co., Ltd., Tokyo, 1991).

[35] L. J. Zhou, M. E. Carrington, G. Kunstatter, and J. Louko, Smooth and sharp creation of a pointlike source for a $(3+1)$-dimensional quantum field, Phys. Rev. D 95, 085007 (2017).

[36] S. Moriguchi, K. Udagawa, and S. Hitotsumatsu, Sugaku Koshiki II, in Japanese (Iwanami Shoten, Tokyo, 1957).

[37] G. B. Arfken and H. J. Weber, Mathematical Methods for Physicists, 6th ed. (Elsevier Academic Press, California, 2005).

[38] S. Moriguchi, K. Udagawa, and S. Hitotsumatsu, Sugaku Koshiki I, in Japanese (Iwanami Shoten, Tokyo, 1957). 